\title{
The earth is flat $(p>0.05)$ : Significance thresholds and the crisis of unreplicable research
}

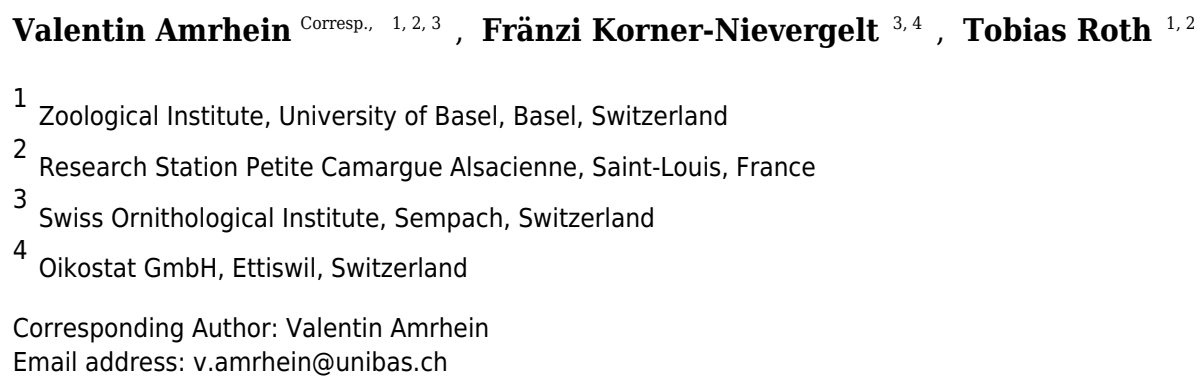

The widespread use of 'statistical significance' as a license for making a claim of a scientific finding leads to considerable distortion of the scientific process (according to the American Statistical Association). We review why degrading p-values into 'significant' and 'nonsignificant' contributes to making studies irreproducible, or to making them seem irreproducible. A major problem is that we tend to take small $p$-values at face value, but mistrust results with larger $p$-values. In either case, $p$-values tell little about reliability of research, because they are hardly replicable even if an alternative hypothesis is true. Also significance $(p \leq 0.05$ ) is hardly replicable: at a good statistical power of $80 \%$, two studies will be 'conflicting', meaning that one is significant and the other is not, in one third of the cases if there is a true effect. A replication can therefore not be interpreted as having failed only because it is nonsignificant. Many apparent replication failures may thus reflect faulty judgment based on significance thresholds rather than a crisis of unreplicable research. Reliable conclusions on replicability and practical importance of a finding can only be drawn using cumulative evidence from multiple independent studies. However, applying significance thresholds makes cumulative knowledge unreliable. One reason is that with anything but ideal statistical power, significant effect sizes will be biased upwards. Interpreting inflated significant results while ignoring nonsignificant results will thus lead to wrong conclusions. But current incentives to hunt for significance lead to selective reporting and to publication bias against nonsignificant findings. Data dredging, p-hacking, and publication bias should be addressed by removing fixed significance thresholds. Consistent with the recommendations of the late Ronald Fisher, p-values should be interpreted as graded measures of the strength of evidence against the null hypothesis. Also larger p-values offer some evidence against the null hypothesis, and they cannot be interpreted as supporting the null hypothesis, falsely concluding that 'there is no effect'. Information on possible true effect sizes that are compatible with the data must be 
obtained from the point estimate, e.g., from a sample average, and from the interval estimate, such as a confidence interval. We review how confusion about interpretation of larger p-values can be traced back to historical disputes among the founders of modern statistics. We further discuss potential arguments against removing significance thresholds, for example that decision rules should rather be more stringent, that sample sizes could decrease, or that $p$-values should better be completely abandoned. We conclude that whatever method of statistical inference we use, dichotomous threshold thinking must give way to non-automated informed judgment. 
1 The earth is flat $(p>0.05)$ : Significance thresholds and the crisis of

2 unreplicable research

3

4

5 Valentin Amrhein ${ }^{1,2,3}$, Fränzi Korner-Nievergelt ${ }^{3,4}$, Tobias Roth ${ }^{1,2}$

6

7

8 'Zoological Institute, University of Basel, Basel, Switzerland

$9{ }^{2}$ Research Station Petite Camargue Alsacienne, Saint-Louis, France

$10{ }^{3}$ Swiss Ornithological Institute, Sempach, Switzerland

$11{ }^{4}$ Oikostat GmbH, Ettiswil, Switzerland

12

13

14 Corresponding Author: Valentin Amrhein

15

16 Email address: v.amrhein@unibas.ch

17 


\section{Abstract}

The widespread use of 'statistical significance' as a license for making a claim of a scientific finding leads to considerable distortion of the scientific process (according to the American Statistical Association). We review why degrading p-values into 'significant' and 'nonsignificant' contributes to making studies irreproducible, or to making them seem irreproducible. A major problem is that we tend to take small $p$-values at face value, but mistrust results with larger pvalues. In either case, p-values tell little about reliability of research, because they are hardly replicable even if an alternative hypothesis is true. Also significance $(\mathrm{p} \leq 0.05)$ is hardly replicable: at a good statistical power of $80 \%$, two studies will be 'conflicting', meaning that one is significant and the other is not, in one third of the cases if there is a true effect. A replication can therefore not be interpreted as having failed only because it is nonsignificant. Many apparent replication failures may thus reflect faulty judgment based on significance thresholds rather than a crisis of unreplicable research. Reliable conclusions on replicability and practical importance of a finding can only be drawn using cumulative evidence from multiple independent studies. However, applying significance thresholds makes cumulative knowledge unreliable. One reason is that with anything but ideal statistical power, significant effect sizes will be biased upwards. Interpreting inflated significant results while ignoring nonsignificant results will thus lead to wrong conclusions. But current incentives to hunt for significance lead to selective reporting and to publication bias against nonsignificant findings. Data dredging, $\mathrm{p}$-hacking, and publication bias should be addressed by removing fixed significance thresholds. Consistent with the recommendations of the late Ronald Fisher, p-values should be interpreted as graded measures of the strength of evidence against the null hypothesis. Also larger p-values offer some evidence against the null hypothesis, and they cannot be interpreted as supporting the null hypothesis, falsely concluding that 'there is no effect'. Information on possible true effect sizes that are compatible with the data must be obtained from the point estimate, e.g., from a sample average, and from the interval estimate, such as a confidence interval. We review how confusion about interpretation of larger p-values can be traced back to historical disputes among the founders of modern statistics. We further discuss potential arguments against removing significance thresholds, for example that decision rules should rather be more stringent, that sample sizes could decrease, or that p-values should better be completely abandoned. We conclude that 
whatever method of statistical inference we use, dichotomous threshold thinking must give way to non-automated informed judgment.

\section{Introduction}

"It seems to me that statistics is often sold as a sort of alchemy that transmutes randomness into certainty, an 'uncertainty laundering' that begins with data and concludes with success as measured by statistical significance. (...) The solution is not to reform p-values or to replace them with some other statistical summary or threshold, but rather to move toward a greater acceptance of uncertainty and embracing of variation."

Andrew Gelman (2016)

Scientific results can be irreproducible for at least six major reasons (Academy of Medical Sciences 2015). There may be (1) technical problems that are specific to the particular study. There may be more general problems like (2) weak experimental design or (3) methods that are not precisely described so that results cannot be reproduced. And there may be statistical issues affecting replicability that are largely the same in many fields of research. Such issues are (4) low statistical power, and (5) 'data dredging' or 'p-hacking' by trying alternative analyses until a significant result is found, which then is selectively reported without mentioning the nonsignificant outcomes. Related to that, (6) publication bias occurs when papers are more likely to be published if they report significant results (Bishop \& Thompson 2016).

Is a major part of an apparent crisis of unreplicable research caused by the way we use statistics for analyzing, interpreting, and communicating our data? Significance testing has been severely criticized for about a century (e.g., Boring 1919; Berkson 1938; Rozeboom 1960; Oakes 1986; Cohen 1994; Ziliak \& McCloskey 2008; Kline 2013), but the prevalence of p-values in the biomedical literature is still increasing (Chavalarias et al. 2016). For this review, we assume that a revolution in applied statistics with the aim of banning p-values is not to be expected nor necessarily useful, and that the main problem is not p-values but how they are used (Gelman 2013b; Gelman 2016). We argue that one of the smallest incremental steps to address statistical issues of replicability, and at the same time a most urgent step, is to remove thresholds of 
80 statistical significance like $\mathrm{p}=0.05$ (see Box 1 ). This may still sound fairly radical to some, but

81 for the following reasons it is actually not.

82 First, p-values can be traditionally employed and interpreted as evidence against null

83 hypotheses also without using a significance threshold. However, what needs to change for

84 reducing data dredging and publication bias is our overconfidence in what significant p-values

85

86

87 can tell, and, as the other side of the coin, our bad attitude towards p-values that do not pass a threshold of significance. As long as we treat our larger p-values as unwanted children, they will continue disappearing in our file drawers, causing publication bias, which has been identified as the possibly most prevalent threat to reliability and replicability of research already a long time ago (Sterling 1959; Wolf 1961; Rosenthal 1979). Still today, in an online survey of 1576 researchers, selective reporting was considered the most important factor contributing to irreproducible research (Baker 2016).

Second, the claim to remove fixed significance thresholds is widely shared among statisticians. In 2016, the American Statistical Association (ASA) published a statement on pvalues, produced by a group of more than two dozen experts (Wasserstein \& Lazar 2016). While there were controversial discussions about many topics, the consensus report of the ASA features the following statement: "The widespread use of 'statistical significance' (generally interpreted as ' $\mathrm{p} \leq 0.05^{\prime}$ ) as a license for making a claim of a scientific finding (or implied truth) leads to considerable distortion of the scientific process" (Wasserstein \& Lazar 2016). And a subgroup of seven ASA statisticians published an extensive review of 25 misinterpretations of p-values, confidence intervals, and power, closing with the words: "We join others in singling out the degradation of p-values into 'significant' and 'nonsignificant' as an especially pernicious statistical practice" (Greenland et al. 2016).

The idea of using p-values not as part of a binary decision rule but as a continuous measure of evidence against the null hypothesis has had many advocates, among them the late Ronald Fisher (Fisher 1956; Fisher 1958; Eysenck 1960; Skipper, Guenther \& Nass 1967; Labovitz 1968; Edgington 1970; Oakes 1986; Rosnow \& Rosenthal 1989; Stoehr 1999; Sterne \& Smith 2001; Gelman 2013a; Greenland \& Poole 2013; Higgs 2013; Savitz 2013; Madden, Shah \& Esker 2015; Drummond 2016; Lemoine et al. 2016; van Helden 2016). Removing significance thresholds was also suggested by authors sincerely defending p-values against their critics (Weinberg 2001; Hurlbert \& Lombardi 2009; Murtaugh 2014a). 
111 In the following, we start with reviewing what p-values can tell about replicability and

112 reliability of results. That this will not be very encouraging should not be taken as another advice

113 to stop using p-values. Rather, we want to stress that reliable information about reliability of

114 results cannot be obtained from p-values nor from any other statistic calculated in individual

115 studies. Instead, we should design, execute, and interpret our research as a prospective meta-

116 analysis' (Ioannidis 2010), to allow combining knowledge from multiple independent studies,

117 each producing results that are as unbiased as possible. Our aim is to show that not p-values, but

118 significance thresholds are a serious obstacle in this regard.

119 We therefore do not focus on general misconceptions about p-values, but on problems with,

120 history of, and solutions for applying significance thresholds. After discussing why significance

121 cannot be used to reliably judge the credibility of results, we review why applying significance

122 thresholds reduces replicability. We then describe how the switch in interpretation that often

123 follows once a significance threshold is crossed leads to proofs of the null hypothesis like 'the

124 earth is flat $(\mathrm{p}>0.05)^{\prime}$. We continue by summarizing opposing recommendations by Ronald

125 Fisher versus Jerzy Neyman and Egon Pearson that led to the unclear status of nonsignificant

126 results, contributing to publication bias. Finally, we outline how to use graded evidence and

127 discuss potential arguments against removing significance thresholds. We conclude that we side

128 with a neoFisherian paradigm of treating p-values as graded evidence against the null hypothesis.

129 We think that little would need to change, but much could be gained by respectfully discharging

130 significance, and by cautiously interpreting p-values as continuous measures of evidence.

Box 1: Significance thresholds and two sorts of reproducibility

Inferential reproducibility might be the most important dimension of reproducibility and "refers to the drawing of qualitatively similar conclusions" from an independent replication of a study (Goodman, Fanelli \& Ioannidis 2016). Some people erroneously conclude that a nonsignificant replication automatically contradicts a significant original study. Others will look at the observed effect, which might hint into the same direction as in the original study, and therefore come to the opposite conclusion. Since judgment based on significance is faulty, 141 judgment based on effect sizes will increase inferential reproducibility. Further, it is current 
142 practice to interpret $p$-values $>0.05$ as evidence either against the null hypothesis, or (falsely) in favor of a null effect, or as no evidence at all. Researchers will increase inferential reproducibility if they refrain from turning their conclusion upside down once a significance threshold is crossed, but instead take the p-value as providing graded evidence against the null hypothesis.

Results reproducibility, or replicability, "refers to obtaining the same results from the conduct of an independent study" (Goodman, Fanelli \& Ioannidis 2016). How results should look like to be considered 'the same', however, remains operationally elusive. What matters, according to Goodman, Fanelli \& Ioannidis (2016), "is not replication defined by the presence or absence of statistical significance, but the evaluation of the cumulative evidence and assessment of whether it is susceptible to major biases." Unfortunately, adhering to significance thresholds brings considerable bias to the published record of cumulative evidence. If results are selected for publication and interpretation because they are significant, conclusions will be invalid. One reason is that the lens of statistical significance usually sees only inflated effects and results that are "too good to be true" (Gelman 2015). Researchers will increase replicability if they report and discuss all results, irrespective of the sizes of their p-values.

\section{P-values are hardly replicable}

In most cases, null hypothesis significance testing is used to examine how compatible some data are with the null hypothesis that the true effect size is zero. The statistical test result is a p-value informing on the probability of the observed data, or data more extreme, given that the null hypothesis is true (and given that all other assumptions about the model are correct; Greenland et al. 2016). If $\mathrm{p} \leq 0.05$, we have learned in our statistics courses to call this significant, to reject the null hypothesis, and to accept an alternative hypothesis about some non-zero effect in the larger population.

170 However, we do not know nor can we infer whether the null hypothesis or an alternative 171 hypothesis is true. On the basis of one single study, it is logically impossible to draw a firm conclusion (Oakes 1986, p. 128), for example because a small p-value either means the null 
173 hypothesis is not true, or else it is true but we happened to find relatively unlikely data. It is for

174 those "possible effects of chance coincidence" that Ronald Fisher wrote: "No isolated

175 experiment, however significant in itself, can suffice for the experimental demonstration of any

176 natural phenomenon" (Fisher 1937, p. 16).

177 Unlike a widespread belief, the p-value itself does not indicate how replicable our results are

178 (Miller 2009; Greenland et al. 2016). We hope that a small p-value means our results are reliable

179 and a replication study would have a good chance to find a small p-value again. Indeed, an

180 extensive research project replicating 100 psychological studies reported that the chance to find a

181 significant result in a replication was higher if the $p$-value in the original study was smaller; but

182 of 63 original studies with $\mathrm{p}<0.02$, only $26(41 \%)$ had $\mathrm{p}<0.05$ in the replication (Open Science

183 Collaboration 2015).

184 Apparently, p-values are hardly replicable. This is most evident if the null hypothesis is true,

185 because then $p$-values are uniformly distributed and thus all values are equally likely to occur

186 (Hung et al. 1997; Boos \& Stefanski 2011; Colquhoun 2014). However, a null hypothesis of an

187 effect of exactly zero is often unlikely to be true (Loftus 1993; Cohen 1994; Stahel 2016). After

188 all, in most cases we did our study because we had some a priori reason to believe that the true

189 effect is not zero.

190 Unfortunately, p-values are highly variable and thus are hardly replicable also if an alternative

191 hypothesis is true, or if the observed effect size is used for calculating the distribution of $p$

192 (Goodman 1992; Senn 2002; Cumming 2008; Halsey et al. 2015). Cumming (2008) showed that

193 if we observe an effect with $\mathrm{p}=0.05$ in a first study, a replication study will find a $\mathrm{p}$-value

194 between 0.00008 and 0.44 with $80 \%$ probability (given by an $80 \%$ 'prediction interval'), and of

$195>0.44$ with $10 \%$ probability. For $\mathrm{p}=0.5$ in a first study, Lazzeroni, Lu \& Belitskaya-Levy (2014)

196 found that $95 \%$ of replication p-values will have sizes between 0.003 and 0.997 (given by a $95 \%$

197 prediction interval).

198 This enormous variability from sample to sample was called the 'dance of the p-values'

199 (Cumming 2012; Cumming 2014). Because the p-value is based upon analysis of random

200 variables, it is a random variable itself, and it behaves as such (Hung et al. 1997; Sackrowitz \&

201 Samuel-Cahn 1999; Murdoch, Tsai \& Adcock 2008). For some reason, however, the stochastic

202 aspect of $\mathrm{p}$-values is usually neglected, and $\mathrm{p}$ is reported as a fixed value without a measure of

203 vagueness or unreliability (Sackrowitz \& Samuel-Cahn 1999; Cumming 2008; Barber \& Ogle 
204 2014). Indeed, we cannot use standard errors or confidence intervals for $p$, because they would

205 estimate unobservable population parameters; and because the p-value is a property of the 206 sample, there is no unobservable 'true p-value' in the larger population (Cumming 2012, p. 133).

207 But as shown, e.g., by Cumming (2008), we could present our p-values with prediction intervals, 208 which are intervals with a specified chance of including the p-value given by a replication.

209 If we would make vagueness of p-values visible by using prediction intervals, it would

210 become immediately apparent that the information content of $\mathrm{p}=0.04$ and of $\mathrm{p}=0.06$ is essentially 211 the same (Dixon 2003; Halsey et al. 2015; Giner-Sorolla 2016), and that "the difference between

212 'significant' and 'not significant' is not itself statistically significant" (Gelman \& Stern 2006). It is 213 a good habit to publish exact $\mathrm{p}$-values rather than uninformative statements like ' $\mathrm{p}>0.05$ '; but 214 additional decimal places and an equal sign should not mislead us to give p-values an aura of 215 exactitude (Boos \& Stefanski 2011; Halsey et al. 2015).

216 P-values are only as reliable as the samples from which they are obtained (Halsey et al. 2015).

217 They inherit their vagueness from the uncertainty of point estimates like the sample average from 218 which they are calculated. But they clearly give less information on uncertainty, reliability or 219 repeatability of the point estimate than is evident from a 95\% confidence interval (which is an $22083 \%$ prediction interval for the point estimate of a replication; Cumming 2014). While the 221 confidence interval measures precision and, therefore, reliability of the point estimate, the p222 value mixes information on the size of the effect and how precisely it was measured. Thus, two 223 point estimates can be equally reliable but may have different effect sizes and therefore different 224 p-values (Fig. 1A, D). And a small p-value can arise because a point estimate is far off the null 225 value or because the sample size is large; but data may still show considerable variation around 226 the point estimate that therefore would not be very reliable (Fig. 1A versus E).

227 So by definition, the p-value reflects our observed evidence against a null hypothesis, but it 228 does not directly measure reliability of the effect that we found in our sample. And we saw that 229 p-values are much more variable than most people think (Lai, Fidler \& Cumming 2012). We 230 therefore must learn to treat $\mathrm{p}$-values like any other descriptive statistic and refrain from taking 231 them at face value when we want to draw inference beyond our particular sample data (Miller 232 2009). Using observed p-values to make a binary decision whether or not to reject a hypothesis is 233 as risky as placing our bets on a sample average without considering that there might be error 
234 attached to it. If p-values are hardly replicable, so too are decisions based on them (Kline 2013, 235 p. 13).

236 It seems that the only way to know how replicable our results are is to actually replicate our 237 results. Science will proceed by combining cumulative knowledge from several studies on a 238 particular topic, summarized for example in meta-analyses (Schmidt 1992; Schmidt 1996;

239 Goodman, Fanelli \& Ioannidis 2016). And one reason why replication studies are rarely done 240 (Kelly 2006) may be that between $37 \%$ and $60 \%$ of academic professionals seem to think the p241 value informs on the probability of replication (Gigerenzer, Krauss \& Vitouch 2004). After all, 242 why actually replicate a study when the p-value gives us virtual replications (Ziliak \& 243 McCloskey 2008, p. 127)?

244 If we do a true replication study, however, our observed p-value will be a realization of a 245 random variable again and will be as unreliable as in the first study. A single replication thus can 246 neither validate nor invalidate the original study (Maxwell, Lau \& Howard 2015; Leek \& Jager 247 2016; Nosek \& Errington 2017). It simply adds a second data point to the larger picture.

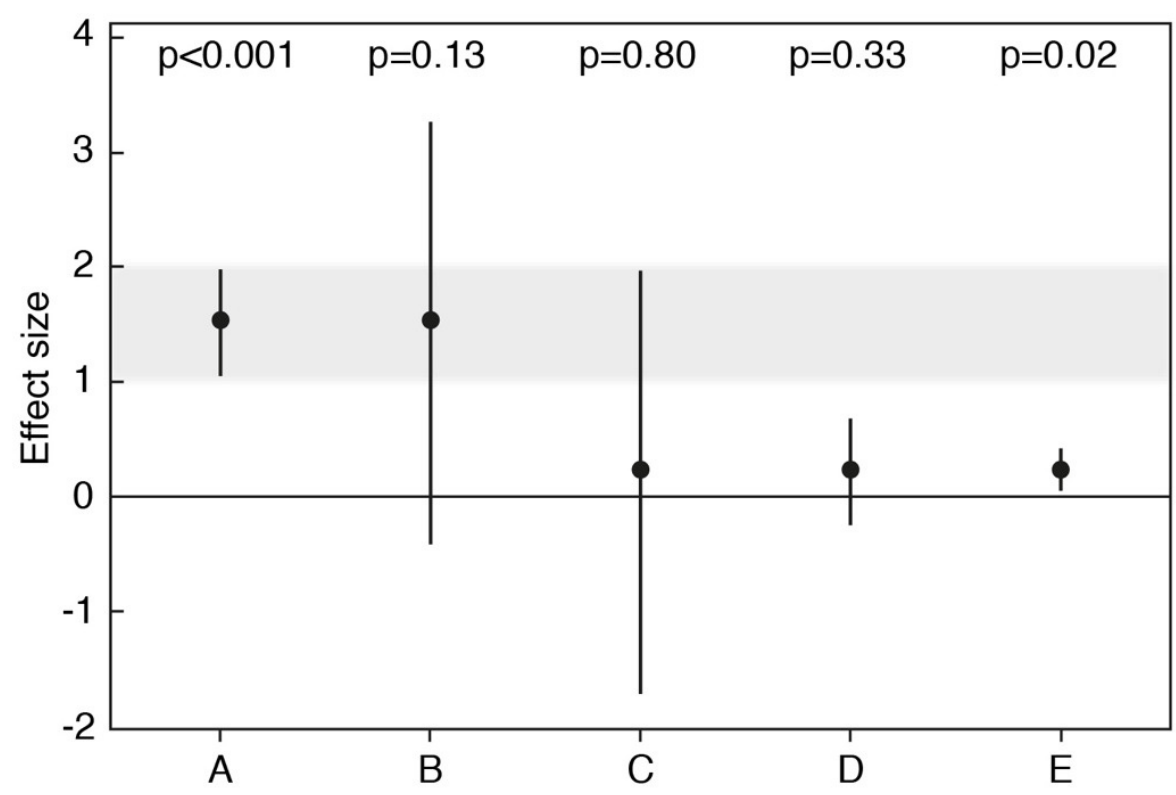

251

252 Figure 1 Averages and $\mathbf{9 5 \%}$ confidence intervals from five simulated studies. P-values are 253 from one sample t-tests, and sample sizes are $\mathrm{n}=30$ each (adapted from Korner-Nievergelt \& 254 Hüppop 2016). Results A and E are relatively incompatible with the null hypothesis that the true 
255 effect size (the population average) is zero. Note that p-values in A versus D, or B versus C, are

256 very different, although the estimates have the same precision and are thus equally reliable. Note

257 also that the $\mathrm{p}$-value in $\mathrm{A}$ is smaller than in $\mathrm{E}$ although variation is larger, because the point

258 estimate in $\mathrm{A}$ is farther off the null value. If we define effect sizes between 1 and 2 as

259 scientifically or practically important, result A is strong evidence that the effect is important, and

260 result $\mathrm{E}$ is clear evidence that the effect is not important, because the small effect size was

261 estimated with high precision. Result B is relatively clear evidence that the effect is not strongly

262 negative and could be important, given that a value close to the center of a $95 \%$ confidence

263 interval is about seven times as likely to be the true population parameter as is a value near a

264 limit of the interval (Cumming 2014). Result $\mathrm{C}$ is only very weak evidence against the null

265 hypothesis, and because plausibility for the parameter is greatest near the point estimate, we may

266 say that the true population average could be relatively close to zero. However, result C also

267 shows why a large p-value cannot be used to 'confirm' or 'support' the null hypothesis: first, the

268 point estimate is larger than zero, thus the null hypothesis of a zero effect is not the hypothesis

269 most compatible with the data. Second, the confidence interval shows population averages that

270 would be consistent with the data and that could be strongly negative, or positive and even

271 practically important. Because of this large uncertainty covering qualitatively very different

272 parameter values, we should refrain from drawing conclusions about practical consequences

273 based on result C. In contrast, result D is only weak evidence against the null hypothesis, but

274 precision is sufficient to infer that possible parameter values are not far off the null and that the

275 effect is practically not important. Result $\mathrm{C}$ is thus a case in which the large $\mathrm{p}$-value and the wide

276 confidence interval roughly say the same, which is that inference is difficult. Results B and D

277 can be meaningfully interpreted even though p-values are relatively large.

278

279

280 Significance is hardly replicable

281

282 There is currently no consensus how replicability or results reproducibility should be measured

283 (see Box 1; Open Science Collaboration 2015; Baker 2016; Goodman, Fanelli \& Ioannidis

284 2016). Whether 'the same results' were obtained may be judged by comparing effect sizes and

285 interval estimates, or by relying on subjective assessment by the scientists performing the 
286 replication (Open Science Collaboration 2015). What counts in the end will be the cumulative

287 evidential weight from multiple independent studies; and those studies will probably show 288 considerable variation in effect sizes (Goodman, Fanelli \& Ioannidis 2016; Patil, Peng \& Leek 289 2016).

290 Traditionally, the success versus failure of a replication is defined in terms of whether an 291 effect in the same direction as in the original study has reached statistical significance again 292 (Miller 2009; Open Science Collaboration 2015; Simonsohn 2015; Fabrigar \& Wegener 2016; 293 Nosek \& Errington 2017). However, p-values are difficult to compare because they are sensitive 294 to many differences among studies that are irrelevant to whether results are in agreement 295 (Greenland et al. 2016, p. 343). For example, even if the point estimates are exactly the same, pvalues of two studies may be on opposite sides of 0.05 because estimation precision or sample sizes differ (Simonsohn 2015). Further, if the p-value itself is hardly replicable, we would be surprised if $\mathrm{p} \leq 0.05$ were replicable.

So how likely will two studies both turn out to be significant? It is sometimes suggested that replicability of significance is given by the statistical power of the test used in the replication study. Power is defined as the probability that a test will be significant given that the alternative hypothesis is true. However, because in real life we do not know whether the alternative hypothesis is true, also power does not help in judging replicability of empirical results. But if we theoretically assume that the alternative hypothesis is true, we can use power to make simple calculations about the probability that $\mathrm{p}$-values cross a significance threshold such as $\mathrm{p}=0.05$.

If two studies on a true alternative hypothesis have a reasonable sample size and thus the recommended statistical power of $80 \%$, the probability that both studies are significant is $80 \%$ * $80 \%=64 \%$. As exemplified by Greenland et al. (2016), this means that under the luckiest circumstances, e.g. when the alternative hypothesis is true, when there is no publication bias, and when statistical power is good, then two studies will both be significant in only $64 \%$ of cases.

311 The probability that one study is significant and the other is not is $(80 \% * 20 \%)+(20 \% * 80 \%)=$ $31232 \%$ (Greenland et al. 2016; 20\% is the beta error of accepting the null hypothesis although it is 313 false, which equals 1 - power). In one third of fairly ideal replications, results will traditionally 314 be interpreted as conflicting, and replication as having failed.

315 However, the above replicabilities of significance are probably overestimated for two reasons.

316 First, replication studies often report smaller effect sizes than the original studies due to 
317 publication bias in the original studies (see below). Second, most studies end up with a power

318 much smaller than $80 \%$. For example, average power to detect medium sized effects was about

$31940-47 \%$ in 10 journals on animal behavior (Jennions \& Møller 2003), and median power in

320 neuroscience was reported to be about 21\% (Button et al. 2013b). In the Journal of Abnormal

321 Psychology, in which Jacob Cohen (1962) found a median power of $46 \%$ for a medium effect in

322 1960, power dropped to $37 \%$ in 1984 (Sedlmeier \& Gigerenzer 1989). As summarized from 44

323 reviews on statistical power in the social, behavioral and biological sciences, average power to

324 detect small effects was 24\% (Smaldino \& McElreath 2016).

325 If we repeat the exercise by Greenland et al. (2016) with a more realistic power of $40 \%$, we

326 obtain a probability that both studies are significant of $40 \% * 40 \%=16 \%$, and a probability that

327 there are conflicting results of $(40 \% * 60 \%)+(60 \% * 40 \%)=48 \%$. This means that even if we

328 did everything right, except for having only about average power, and if there is a true effect in

329 the larger population, about half of our replications will fail by traditional significance standards

330 (i.e., one study is significant and the other is not). And only about one in six studies will

331 significantly replicate the significant result of another study.

332 This is of course not the fault of the p-value. It is the fault of us defining replication success as

333 the event of crossing a significance threshold. As Greenland et al. (2016) put it, "one could

334 anticipate a 'replication crisis' even if there were no publication or reporting bias, simply because

335 current design and testing conventions treat individual study results as dichotomous outputs of

336 significant/nonsignificant or reject/accept". Even in ideal replication studies, significance as

337 defined by classical thresholds is not to be expected, and nonsignificance cannot be used as a

338 criterion to undermine the credibility of a preceding study (Goodman, Fanelli \& Ioannidis 2016).

339

340 Significance thresholds reduce replicability

341

342 In fact, it would be highly dubious if replication success in terms of statistical significance were

343 larger than just described. This would indicate that researchers suppress nonsignificant

344 replications and selectively report significant outcomes, and that there is publication bias against

345 nonsignificant studies (Francis 2013). However, when nonsignificant results on a particular

346 hypothesis remain unpublished, any significant support for the same hypothesis is rendered

347 essentially uninterpretable (ASA statement; Wasserstein \& Lazar 2016). If white swans remain 
348 unpublished, reports of black swans cannot be used to infer on general swan color. In the worst 349 case, publication bias means according to Rosenthal (1979) that the 95\% of studies that correctly 350 yield nonsignificant results may be vanishing in file drawers, while journals may be filled with 351 the $5 \%$ of studies committing the alpha error by claiming to have found a significant effect when 352 in reality the null hypothesis is true.

353 However, selective reporting was encouraged since the early days of significance testing. As 354 Ronald Fisher (1937, p. 15) wrote, "it is usual and convenient for experimenters to take 5 per

355

356

357

358

359

360

361

362

363

364

365

366

367

368

369

370

371

372

373

374

375

376

377

378 cent as a standard level of significance, in the sense that they are prepared to ignore all results which fail to reach this standard", an advice he gave at least since 1926 (Fisher 1926). As can be read in guidelines on writing papers and theses, students should "expect that you will produce many more figures and perform many more statistical tests than will be included in the final written product" (Lertzman 1995). Based on a survey of over 2000 psychologists, John, Loewenstein \& Prelec (2012) estimated that among researchers, the prevalence of having engaged in selective reporting of studies that 'worked' or of only a subset of measured variables is $100 \%$.

No wonder that a bias towards publishing significant results was observed for a long time and in many research areas (Sterling 1959; Csada, James \& Espie 1996; Gerber \& Malhotra 2008; Song et al. 2010; Dwan et al. 2013). Today, so-called negative results may disappear from many disciplines and countries (Fanelli 2012; but see de Winter \& Dodou 2015), threatening the reliability of our scientific conclusions and contributing to the crisis of unreplicable research (Gelman 2015). No outright fraud, no technical fault or bad experimental design are necessary to render a study irreproducible; it is sufficient that we report results preferentially if they cross a threshold of significance.

The problem with selective reporting becomes even worse because significant results are not a random sample from all possible results - significant results are biased results. If a number of studies are done on a population with a fixed effect size, studies that due to sampling variation find a larger effect are more likely to be significant than those that happen to find smaller effects (Schmidt 1992). Using statistical significance as a guideline thus typically selects for large effects that are "too good to be true" (Gelman 2015). The consequence is that "most discovered true associations are inflated" (Ioannidis 2008). This effect was called 'truth inflation' (Reinhart 2015), or 'winner's curse' (Zöllner \& Pritchard 2007; Young, Ioannidis \& Al-Ubaydi 2008; 
379 Button et al. 2013b) after how the term is used in economics: in high-risk situations with

380 competitive bidding, the winner tends to be the bidder who most strongly overestimates the value

381 of an object being auctioned (Capen, Clapp \& Campbell 1971; Foreman \& Murnighan 1996).

382 The inflation of effect sizes in significant results declines as statistical power increases (for

383 example because sample sizes are large), and inflation becomes negligible as power approaches

384 100\% (Colquhoun 2014; Gelman \& Carlin 2014; Lemoine et al. 2016). One way to see how this

385 works is to imagine that with a power of $100 \%$, every test becomes significant given that the

386 alternative hypothesis is true; thus, decisions for reporting based on significance would no longer

387 select for large effects, because every effect from every random sample would be significant.

388 However, with a more realistic power of, e.g., $40 \%$, only inflated effects that on average are

389 about 1.5 times larger than the true effect size may cross the significance threshold (Colquhoun

390 2014, Fig. 7).

391 In the ecological and neurological studies summarized in Lemoine et al. (2016) and Button et

392 al. (2013b), sample sizes required to minimize inflation of effect sizes were $n>100$. However, as

393 Lemoine et al. (2016) note, increased sample sizes can only partially offset the problem of

394 inflated effect sizes, because a power near 100\% will usually not be obtained.

395 Selective reporting of inflated significant effects while ignoring smaller and nonsignificant

396 effects will lead to wrong conclusions in meta-analyses synthesizing effect sizes from a larger

397 number of studies (Ferguson \& Heene 2012; van Assen et al. 2014). This is one of the reasons

398 why reliance on significance testing has been accused of systematically retarding the

399 development of cumulative knowledge (Schmidt 1996; Branch 2014).

400 Of course, selective reporting of significant results leads to inflated effects not only in meta-

401 analyses but in every single study. Even in cases in which authors report all conducted tests

402 regardless of their p-values, but then select what to interpret and to discuss based on significance

403 thresholds, the effects from which the authors draw their conclusions will be biased upwards.

404 The problem arises not only by consciously discarding nonsignificant findings. Also largely

405 automated selection procedures may produce inflated effects, for example if genome-wide

406 association studies select findings based on significance thresholds (Göring, Terwilliger \&

407 Blangero 2001; Garner 2007). In statistical model simplification, or model selection, significant

408 predictors will have inflated point estimates (Whittingham et al. 2006; Ioannidis 2008; 
409 Forstmeier \& Schielzeth 2011), and defining the importance of a predictor variable based on

410 statistical significance will thus lead to distorted results.

411

\section{Truth inflation}

413

414 The p-value can be seen as a measure of surprise (Greenwald et al. 1996): the smaller it is, the 415 more surprising the results are if the null hypothesis is true (Reinhart 2015, p. 9). If one wants to 416 determine which patterns are unusual enough to warrant further investigation, $p$-values are thus 417 perfectly suitable as explorative tools for selecting the largest effects. Whoever is interested in 418 describing the average state of a system, however, should not "choose what to present based on 419 statistical results", because "valid interpretation of those results is severely compromised" unless 420 all tests that were done are disclosed (ASA statement, Wasserstein \& Lazar 2016). And, we 421 might add, unless all results are used for interpretation and for drawing conclusions, irrespective 422 of their p-values.

423 Of course, current incentives lead to 'significance chasing' (Ioannidis 2010) rather than to 424 publishing nonsignificant results. To put it more bluntly, "research is perverted to a hunt for 425 statistically significant results" (Stahel 2016). The road to success is nicely summarized in the 426 online author guidelines of the journal 'Nature' (accessed 2017): "The criteria for a paper to be 427 sent for peer-review are that the results seem novel, arresting (illuminating, unexpected or 428 surprising)." And the p-value, as a measure of surprise, seems to be a great selection tool for that purpose. However, the urge for large effect sizes in novel fields with little prior research is a "perfect combination for chronic truth inflation" (Reinhart 2015, p. 25). As wrote Ioannidis (2008), "at the time of first postulated discovery, we usually cannot tell whether an association exists at all, let alone judge its effect size."

433 Indeed, the strength of evidence for a particular hypothesis usually declines over time, with 434 replication studies presenting smaller effects than original studies (Jennions \& Møller 2002; 435 Brembs, Button \& Munafo 2013; Open Science Collaboration 2015). The reproducibility project 436 on 100 psychological studies showed that larger original effect sizes were associated with greater 437 effect size differences between original and replication, and that "surprising effects were less 438 reproducible" (Open Science Collaboration 2015). 
Small, early, and highly cited studies tend to overestimate effects (Fanelli, Costas \& Ioannidis

440

441

442

443

444

445

446

447

448

449

450

451

452

453

454

455

456

457

458

459

460

461

462

463

464

465

466

467

468

469 2017). Pioneer studies with inflated effects often appear in higher-impact journals, while studies in lower-impact journals apparently tend to report more accurate estimates of effect sizes (Ioannidis 2005; Munafo, Stothart \& Flint 2009; Munafo \& Flint 2010; Siontis, Evangelou \& Ioannidis 2011; Brembs, Button \& Munafo 2013). The problem is likely publication bias towards significant and inflated effects particularly in the early stages of a potential discovery. At a later time, authors of replication studies might then want, or be allowed by editors, to report results also if they found only negligible or contradictory effects, because such results find a receptive audience in a critical scientific discussion (Jennions \& Møller 2002). Replications therefore tend to suffer less from publication bias than original studies (Open Science Collaboration 2015).

So far, academic reward mechanisms often focus on statistical significance and newsworthiness of results rather than on reproducibility (Ioannidis et al. 2014). Also journalists and media consumers and, therefore, all of us ask for the novel, unexpected and surprising. Thus the average truth often does not make it to the paper and the public, and much of our attention is attracted by exaggerated results.

\section{The earth is flat $(p>0.05)$}

The average truth might be nonsignificant and non-surprising. But this does not mean the truth equals zero. In the last decades, many authors have compiled lists with misinterpretations regarding the meaning of the p-value (e.g., Greenland et al. 2016), and surveys showed that such false beliefs are widely shared among researchers (Oakes 1986; Lecoutre, Poitevineau \& Lecoutre 2003; Gigerenzer, Krauss \& Vitouch 2004; Badenes-Ribera et al. 2016). The "most devastating" of all false beliefs is probably that "if a difference or relation is not statistically significant, then it is zero, or at least so small that it can safely be considered to be zero" (Schmidt 1996). For example, if two studies are called conflicting or inconsistent because one is significant and the other is not, it may be implicitly assumed that the nonsignificant effect size was zero (Cumming 2012, p. 31).

Jacob Cohen (1994) published his classic critique of the use of significance tests under the title "The earth is round $(\mathrm{p}<.05)$ ". What if this test happens to be nonsignificant? In $38 \%$ to $63 \%$ of articles sampled from five journals of psychology, neuropsychology and conservation biology, 
470 nonsignificant results were interpreted as 'there is no effect', which means that a null hypothesis 471 was accepted or 'proven' (Finch, Cumming \& Thomason 2001; Schatz et al. 2005; Fidler et al. 472 2006; Hoekstra et al. 2006).

473 In Cohen's example, 'no effect' would probably mean 'the earth is flat ( $p>0.05)$.' And this is 474 not far from reality. Similar cases abound in the published literature, such as "lamb kill was not 475 correlated to trapper hours $\left(r_{12}=0.50, P=0.095\right) "$ (cited in Johnson 1999). It may be completely 476 obvious that the null hypothesis of 'no effect' cannot be true, as judging from a large but 477 nonsignificant correlation coefficient, from clear but nonsignificant differences between averages 478 in a figure, or from common sense; but still we do not hesitate to apply our "binary thinking, in 479 which effects and comparisons are either treated as zero or are treated as real" (Gelman 2013b). 480 How is this possible, since "of course, everyone knows that one can't actually prove null 481 hypotheses" (Cohen 1990)?

482 We probably tend to misinterpret p-values because significance testing "does not tell us what 483 we want to know, and we so much want to know what we want to know that, out of desperation, 484 we nevertheless believe that it does" (Cohen 1994). Null hypothesis significance testing is not 485 about estimating the probability that the null hypothesis or the alternative hypothesis is true 486 such a claim would be reserved to Bayesian testers, and even they would not be able to 'prove' 487 any hypothesis. Null hypothesis testing is about the probability of our data given that the null 488 hypothesis is true.

489 The problem is "the researcher's 'Bayesian' desire for probabilities of hypotheses" (Gigerenzer 490 1993). It may be hopeless to temper this desire, since also Fisher himself held a "quasi-Bayesian 491 view that the exact level of significance somehow measures the confidence we should have that 492 the null hypothesis is false" (Gigerenzer 1993). Yet, Fisher seemed to be clear about proving the 493 null: a hypothesis cannot be "proved to be true, merely because it is not contradicted by the 494 available facts" (Fisher 1935). And, therefore, "it is a fallacy, so well known as to be a standard 495 example, to conclude from a test of significance that the null hypothesis is thereby established; at most it may be said to be confirmed or strengthened" (Fisher 1955; italics in original).

497 The last sentence, however, shows that also Fisher vacillated (Gigerenzer et al. 1989, p. 97). 498 In fact, the null hypothesis cannot be confirmed nor strengthened, because very likely there are many better hypotheses: "Any p-value less than 1 implies that the test [null] hypothesis is not the hypothesis most compatible with the data, because any other hypothesis with a larger $p$-value 
501 would be even more compatible with the data" (Greenland et al. 2016). This can be seen when

502 looking at a 'nonsignificant' 95\% confidence interval that encompasses not only zero but also

503 many other null hypotheses that would be compatible with the data, or, in other words, that

504 would not be rejected using a 5\% threshold (Fig. 1C; Tukey 1991; Tryon 2001; Hoekstra,

505 Johnson \& Kiers 2012). Within the confidence interval, zero is usually not the value that is

506 closest to the observed point estimate. And even if the point estimate is exactly zero and thus

$507 \mathrm{p}=1$, there will be many other hypotheses [i.e., the values covered by the confidence interval]

508 that are highly consistent with the data, so that a definitive conclusion of 'no association' cannot

509 be deduced from a p-value, no matter how large" (Greenland et al. 2016).

510

511 "Limbo of suspended disbelief"

512

513 It is easy to imagine research in which falsely claiming a true null effect causes great harm. To

514 give a drastic example, Ziliak \& McCloskey (2008, p. 28) cite a clinical trial on the painkiller

515 Vioxx that reports data on heart attacks and other adverse events (Lisse et al. 2003). Lisse and

516 colleagues found several ' $\mathrm{p}>0.2$ ', among them that "the rofecoxib ['Vioxx'] and naproxen [generic

517 drug] groups did not differ significantly in the number of thrombotic cardiovascular events (...)

518 (10 vs. 7; $\mathrm{P}>0.2)$ ". The conclusion was that "the results demonstrated no difference between

519 rofecoxib and naproxen." Later, the unjustified proof of the null caused more suffering, and the

520 manufacturer Merck took Vioxx off the market and faced more than 4200 lawsuits by August 20,

5212005 (Ziliak \& McCloskey 2008).

522 Unfortunately, if we finally accept that we cannot accept a null hypothesis, obtaining a

523 nonsignificant result becomes downright annoying. If the null hypothesis cannot be rejected

524 because $\mathrm{p}>0.05$, it is often recommended to 'suspend judgment' (Tryon 2001; Hurlbert \&

525 Lombardi 2009), which leaves the null hypothesis "in a kind of limbo of suspended disbelief"

526 (Edwards, Lindman \& Savage 1963). As Cohen (1990) put it, "all you could conclude is that you

527 couldn't conclude that the null was false. In other words, you could hardly conclude anything."

528 This unfortunate state becomes even worse because usually the researchers are blamed for not

529 having collected more data. Indeed, in correlational research, most null hypotheses of an effect

530 of exactly zero are likely wrong at least to a small degree (Edwards, Lindman \& Savage 1963;

531 Meehl 1967; Delong \& Lang 1992; Lecoutre \& Poitevineau 2014 - but see Hagen 1997; Hagen 
532 1998; Thompson 1998; Krueger 2001 for a critical discussion). Therefore, most tests would 533 probably produce significant results if only one had large enough sample sizes (Oakes 1986;

534 Cohen 1990; Cohen 1994; Gill 1999; Stahel 2016). In other words, a significance test often does

535 not make a clear statement about an effect, but instead it "examines if the sample size is large

536 enough to detect the effect" (Stahel 2016). And because "you can pretty much always get

537 statistical significance if you look hard enough" (Gelman 2015), you were probably "not trying

538 hard enough to find significant results" (Ferguson \& Heene 2012). Nonsignificance therefore

539 seems to be regarded as "the sign of a badly conducted experiment" (Gigerenzer et al. 1989, p.

540 107).

541 As an outgoing editor of a major psychological journal wrote, "the [false] decision not to

542 reject the null hypothesis can be a function of lack of power, lack of validity for the measures,

543 unreliable measurement, lack of experiment control, and so on" (Campbell 1982). Surely all of

544 those influences could also lead to falsely claiming a significant outcome, but the editor

545 concludes: "it is true that there is an evaluation asymmetry between significant and

546 nonsignificant results."

547 Naturally, we develop an aversion to 'null results' and fail to publish them (Ferguson \& Heene

548 2012). Instead, we engage in significance chasing that may promote harmful practices like

549 excluding variables or data with unwanted effects on p-values, stopping to collect data after

550 looking at preliminary tests, rounding down p-values that are slightly larger than 0.05 , or even

551 falsifying data (non-exhaustive list after the survey by John, Loewenstein \& Prelec 2012).

552 Although it is now widely discussed that significant results may be much less reliable than we

553 used to believe, mistrust in nonsignificant results still seems larger than mistrust in significant

554 results, leading to publication bias, which in turn causes significant results to be less reliable.

555 What could be done?

556

557 Accepting history

558

559 First, we should briefly review the rise of modern statistics that started in the 1920s and 1930s.

560 We think that problems like the unclear status of nonsignificant $p$-values have their roots in

561 history, and that understanding those roots will help finding a future role for $\mathrm{p}$-values (for more 
562 details, see Gigerenzer et al. 1989; Gill 1999; Salsburg 2001; Lenhard 2006; Hurlbert \&

563 Lombardi 2009; Lehmann 2011).

564 The three most influential founding fathers, Ronald A. Fisher, Jerzy Neyman and Egon S.

565 Pearson, disagreed on many things, but they agreed that scientific inference should not be made

566 mechanically (Gigerenzer \& Marewski 2015). In their 'hypothesis tests', Neyman and Pearson

567 confronted a point null hypothesis with a point alternative hypothesis. Based on this scenario

568 they discovered alpha and beta errors as well as statistical power. Neyman and Pearson did not

569 request to report p-values, but to make decisions based on predefined alpha and beta errors. They

570 never recommended a fixed significance threshold (Lehmann 2011, p. 55), but rather held that

571 defining error rates "must be left to the investigator" (Neyman \& Pearson 1933a, p. 296).

572 Some years earlier, Fisher had introduced 'significance tests' using p-values on single null

573 hypotheses, and he generally opposed the consideration of alternative hypotheses and of power

574 (Lehmann 2011, p. 51). Fisher did not invent the p-value, which he called 'level of significance',

575 but he was the first to outline formally the logic of its use (Goodman 1993). In 1925, he defined

576 a threshold of $\mathrm{p}=0.05$, based on the proportion of the area under a normal distribution that falls

577 outside of roughly two standard deviations from the mean: "The value for which $\mathrm{P}=\cdot .05$, or 1 in

57820 , is 1.96 or nearly 2; it is convenient to take this point as a limit in judging whether a deviation

579 is to be considered significant or not. Deviations exceeding twice the standard deviation are thus

580 formally regarded as significant" (Fisher 1925). The choice of 0.05 was acknowledged to be

581 "arbitrary" by Fisher (1929, p. 191) and was influenced by earlier definitions of significance by

582 William Gosset, Karl Pearson (the father of Egon) and others (discussed in Cowles \& Davis

583 1982b; Sauley \& Bedeian 1989; Hurlbert \& Lombardi 2009). A main reason why 0.05 was

584 selected and still persists today may be that it fits our subjective feeling that events that happen at

585 least as rarely as $10 \%$ or $1 \%$ of the time are suspiciously unlikely (Cowles \& Davis 1982a; Weiss

586 2011).

587 Throughout his life, Fisher used the p-value mainly to determine whether a result was

588 statistically significant (Lehmann 2011, p. 53). In his last new book, however, Fisher famously

589 wrote that "no scientific worker has a fixed level of significance at which from year to year, and

590 in all circumstances, he rejects hypotheses; he rather gives his mind to each particular case in the

591 light of his evidence and his ideas" (Fisher 1956, p. 42). In the thirteenth edition of "Statistical

592 methods for research workers" (Fisher 1958), he then stated that "the actual value of P (...) 
593 indicates the strength of the evidence against the hypothesis" (p. 80) and that "tests of

594 significance are used as an aid to judgment, and should not be confused with automatic 595 acceptance tests, or 'decision functions' " (p. 128).

596 A main point of controversy was 'inductive inference' that was central to Fisher's thinking

597 (Lehmann 2011, p. 90). Fisher believed that significance tests allow drawing inference from 598 observations to hypotheses, or from the sample to the population (although deducing the 599 probability of data given that a null hypothesis is true may actually look more like deductive 600 inference, from the population to the sample; Thompson 1999).

601 In contrast, Neyman and Pearson thought that inductive inference is not possible in statistical 602 analyses on single studies: "As far as a particular hypothesis is concerned, no test based upon a 603 theory of probability can by itself provide any valuable evidence of the truth or falsehood of that 604 hypothesis" (Neyman \& Pearson 1933a, p. 291). Their hypothesis test was therefore not meant to 605 give a measure of evidence (Goodman 1993), but to provide "rules to govern our behavior" with 606 regard to our hypotheses, to insure that "in the long run of experience, we shall not be too often 607 wrong" (Neyman \& Pearson 1933a, p. 291). The Neyman-Pearson decision procedure was particularly suitable for industrial quality control, or "sampling tests laid down in commercial specifications" (Neyman \& Pearson 1933b). Here, the quality of production may be tested very 610 often over long periods of time, so that a frequentist 'long run of experience' is indeed possible, 611 and manufacturers may indeed require thresholds to decide when to stop the production and to 612 look for the cause of a quality problem (Gigerenzer et al. 1989, p. 100).

613 Applying Neyman-Pearson decision rules means accepting the null hypothesis while rejecting

614 the alternative hypothesis, or vice versa. And Neyman and Pearson did indeed use the word 615 'accept' (e.g., Neyman \& Pearson 1933b). Today, we may still speak about accepting a null 616 hypothesis when it is false as committing a beta error, or error of the second kind. But was it not 617 said that null hypotheses cannot be accepted? Fisher wanted 'inductive inference', and for 618 drawing scientific conclusions, a nonsignificant result can never mean to accept a null 619 hypothesis: "errors of the second kind are committed only by those who misunderstand the 620 nature and application of tests of significance" (Fisher 1935). But Neyman and Pearson invented

621 'inductive behavior' explicitly for avoiding inductive inference. And for a behavioral decision, it 622 is of course possible to accept any kind of premises: "As Neyman emphasized, to accept a 
623 hypothesis is not to regard it as true, or to believe it. At most it means to act as if it were true" 624 (Gigerenzer et al. 1989, p. 101).

625 Thus accepting null hypotheses was encouraged by half of the founding schools of modern 626 statistics. No wonder that "in approximately half of all cases, authors interpret their 627 nonsignificant results as evidence that the null hypothesis is true" (McCarthy 2007, p. 43).

628 Our current null hypothesis significance tests are anonymous hybrids mixing elements both 629 from the Fisherian and the Neyman-Pearson concepts. But mixing two essentially incompatible 630 approaches, one for measuring evidence and the other for making behavioral decisions, of course 631 creates all sorts of problems (Gigerenzer 1993; Goodman 1993; Gill 1999; Hubbard \& Bayarri 632 2003; Schneider 2015). We often use Neyman-Pearson to refer to statistical power and to the two 633 kinds of error. But then in practice we follow Fisher by refusing to specify a concrete point 634 alternative hypothesis, and by interpreting exact p-values as graded measures of the strength of 635 evidence against the null hypothesis (Mundry 2011; Cumming 2012, p. 25). However, we only 636 consistently interpret p-values as strength of evidence as long as they are smaller than 0.05 . For 637 p-values between 0.05 and 0.1 , some authors are willing to acknowledge a statistical 'trend', 638 while others are not. For even larger p-values, we often switch back to a kind of Neyman639 Pearson decision making, which offers no positive inference but seems to allow at least 640 accepting the null hypothesis.

641 It looks like our mistrust in nonsignificant results that leads to publication bias is caused by 642 confusion about interpretation of larger p-values that goes back to historical disputes among the 643 founders of modern statistics.

644

\section{Removing significance thresholds}

647 What could be done? We could again define what a p-value means: the probability of the 648 observed data, or data more extreme, given that the null hypothesis is true. According to the 649 interpretation by the ASA (Wasserstein \& Lazar 2016), smaller p-values cast more, and larger p650 values cast less doubt on the null hypothesis. If we apply those definitions, it falls naturally to 651 take p-values as graded evidence. We should try and forget our black-and-white thresholds 652 (Tukey 1991) and instead consider the p-value as a continuous measure of the compatibility 
653 between the data and the null hypothesis, "ranging from 0 for complete incompatibility to 1 for 654 perfect compatibility" (Greenland et al. 2016).

655 We need to move away from Fisher's early recommendation to ignore nonsignificant results, 656 because following this rule leads to publication bias and to reported effects that are biased 657 upwards. We need to move away from the Neyman-Pearson reject/accept procedure, because it 658 leads to proofs of the null like 'not correlated $(\mathrm{p}=0.095)$ '. Instead, we should listen to the ASA659 statisticians who say that if the "p-value is less than 1, some association must be present in the 660 data, and one must look at the point estimate to determine the effect size most compatible with 661 the data under the assumed model" (Greenland et al. 2016).

662 We are thus encouraged to interpret our point estimate as "our best bet for what we want to 663 know" (Cumming 2007). According to the central limit theorem, sample averages are 664 approximately normally distributed, so with repeated sampling most of them would cluster 665 around the true population average. Within a 95\% confidence interval, the sample average (the 666 point estimate) is therefore about seven times as plausible, or seven times as good a bet for the 667 true population average, as are the limits of the confidence interval (Cumming 2012, p. 99; 668 Cumming 2014).

669 After looking at the point estimate, we should then interpret the upper and lower limits of the 670 confidence interval, which indicate values that are still plausible for the true population 671 parameter. Those values should not appear completely unrealistic or be qualitatively very 672 different; otherwise the width of our confidence interval suggests our estimate is so noisy that we 673 should refrain from drawing firm conclusions about practical consequences (Fig. 1C).

674 If necessary, we should then focus on the p-value as a continuous measure of compatibility 675 (Greenland et al. 2016), and interpret larger p-values as perhaps less convincing but generally 676 'positive' evidence against the null hypothesis, instead of evidence that is either 'negative' or 677 uninterpretable or that only shows we did not collect enough data. In short, we should develop a 678 critical but positive attitude towards larger p-values. This alone could lead to less proofs of the 679 null hypothesis, to less significance chasing, less data dredging, less p-hacking, and ultimately to 680 less publication bias, less inflated effect sizes and more reliable research.

681

And removing significance thresholds is one of the smallest steps that we could imagine to 682 address issues of replicability. Using p-values as graded evidence would not require a change in 683 statistical methods. It would require a slight change in interpretation of results that would be 
684 consistent with the recommendations by the late Ronald Fisher and thus with a neoFisherian 685 paradigm described by Hurlbert \& Lombardi (2009). A difference to Hurlbert \& Lombardi 686 (2009) is that for larger p-values we do not propose 'suspending judgment', which we believe 687 would contribute to selective reporting and publication bias because we usually do not want to 688 report results without evaluating possible conclusions. Instead, we recommend "suspending firm 689 decisions (i.e., interpreting results with extra caution)" (Greenland \& Poole 2013). As we saw, 690 some results can be meaningfully interpreted although their p-values are relatively large (Fig. 691 1B, D); and even if p-values were small, firm decisions would not be possible based on an 692 isolated experiment (Fisher 1937, p. 16).

693

694 For our next scientific enterprise using frequentist statistics, we suggest that we 695

696 a) do our study and our analysis as planned

697 b) report our point estimate, interpret our effect size

698 c) report and interpret an interval estimate, e.g. a 95\% confidence interval

699 d) report the exact p-value

700 e) do not use the word 'significant' and do not deny our observed effect if the p-value is relatively 701 large

702 f) discuss how strong we judge the evidence, and how practically important the effect is.

703

Do we need a scale for interpreting the strength of evidence against the null hypothesis?

705 Graded evidence means there are no thresholds to switch from 'strong' to 'moderate' to 'weak' 706 evidence (Sterne \& Smith 2001). It means that "similar data should provide similar evidence" 707 (Dixon 2003), because "surely, God loves the .06 nearly as much as the .05" (Rosnow \& 708 Rosenthal 1989).

709 There are no 'few-sizes-fit-all' grades of evidence. Instead of following the same decision 710 rules, no matter how large the sample size or how serious we judge measurement error, we 711 should "move toward a greater acceptance of uncertainty and embracing of variation" (Gelman 712 2016). If we have obtained a small p-value, we must be aware of the large variability of p-values, 713 keep in mind that our evidence against the null hypothesis might not be as strong as it seems, and 714 acknowledge that our point estimate is probably biased upwards. If we have obtained a large p- 
715 value, we must be even more aware that many other hypotheses are compatible with our data,

716 including the null hypothesis. Looking at the values covered by the confidence interval will help

717 identifying those competing hypotheses. Very likely there are hypotheses compatible with the

718 data that would cause even greater concern than a zero effect, e.g. if the effect would be in the

719 opposite direction (Fig. 1C) or be much smaller or larger than what we observed in our point

720 estimate. Note that the true effect can also be much smaller or larger than our point estimate if

721 the p-value is small.

722 When discussing our results, we should "bring many contextual factors into play to derive

723 scientific inferences, including the design of a study, the quality of the measurements, the

724 external evidence for the phenomenon under study, and the validity of assumptions that underlie

725 the data analysis" (ASA statement; Wasserstein \& Lazar 2016). For example, results from

726 exploratory studies are usually less reliable than from confirmatory (replication) studies also if

727 their p-values were the same, because exploratory research offers more degrees of freedom in

728 data collection and analysis (Gelman \& Loken 2014; Higginson \& Munafo 2016; Lew 2016).

729 Already half a century ago, Labovitz (1968) compiled a list of criteria for evaluating evidence

730 from p-values that include the practical consequences (costs) of a conclusion, the plausibility of

731 alternative hypotheses, or the robustness of the test.

732 And we must keep in mind that support for our hypothesis will require not just one, but many

733 more independent replications. If those replications do not find the same results, this is not

734 necessarily a crisis, but a natural process by which science proceeds. In most research disciplines

735 in which results are subject to a substantial random error, "the paradigm of accumulating

736 evidence might be more appropriate than any binary criteria for successful or unsuccessful

737 replication" (Goodman, Fanelli \& Ioannidis 2016). Thus, "the replication crisis perhaps exists

738 only for those who do not view research through the lens of meta-analysis" (Stanley \& Spence

739 2014).

740 We therefore need to publish as many of our results as possible, as long as we judge them

741 sufficiently reliable to be reported to the scientific community. And we should publish our

742 results also if we find them neither novel nor arresting, illuminating, unexpected or surprising.

743 Those results that we find trustworthy are the best approximates of reality, especially if they look

744 familiar and expected and replicate what we already think we know. 


\section{Increasing inferential reproducibility}

747

748 Of course, people and journals who want to publish only small p-values will probably continue publishing only small p-values. Perhaps, "forced to focus their conclusions on continuous measures of evidence, scientists would selectively report continuous measures of evidence" (Simonsohn 2014). We hope, however, that bidding farewell to significance thresholds will free at least some of our negative results by allowing them to be positive. And we think the chances that this will happen are good: because it is already happening.

People seem prepared to interpret $p$-values as graded evidence. When asked about the degree of confidence that an experimental treatment really has an effect, the average researcher's confidence was found to drop quite sharply as p-values rose to about 0.1 , but then confidence levelled off until 0.9, essentially showing a graded response (Poitevineau \& Lecoutre 2001). Nuzzo (2015) cites Matthew Hankins, who has collected "more than 500 creative phrases that researchers use to convince readers that their nonsignificant results are worthy of attention (see go.nature.com/pwctoq). These include 'flirting with conventional levels of significance ( $\mathrm{p}>0.1)^{\prime}$, 'on the very fringes of significance $(p=0.099)$ ' and 'not absolutely significant but very probably so $(\mathrm{p}>0.05)^{\prime} . "$

As Hurlbert \& Lombardi (2009) note, even Jerzy Neyman (1977, p. 112) labelled p-values of $0.09,0.03$, and $<0.01$ reported in an earlier paper (Lovasich et al. 1971, table 1, right column) as 'approximately significant', 'significant', and 'highly significant', respectively. And creativity is increasing. Pritschet, Powell \& Horne (2016) searched over 1500 papers in three journals of psychology for terms like 'marginally significant' or 'approaching significance' that were used for p-values between 0.05 to 0.1 and up to 0.18 . They found that "the odds of an article describing a p-value as marginally significant in 2010 were 3.6 times those of an article published in 1970." In 2000 and 2010, the proportion of articles describing at least one p-value as marginally significant were $59 \%$ and $54 \%$.

The rules how to label results if $\mathrm{p}>0.05$ are usually unwritten (Pritschet, Powell \& Horne 2016), so it is current practice to interpret p-values between 0.05 and 0.1 , or even larger p-values, as evidence either against the null hypothesis, or (falsely) in favor of a null effect, or as no evidence at all. This anarchical state of affairs undermines 'inferential reproducibility', which might be the most important dimension of reproducibility and "refers to the drawing of 
777 qualitatively similar conclusions from either an independent replication of a study or a reanalysis

778 of the original study" (Goodman, Fanelli \& Ioannidis 2016; italics supplied). Interpreting larger

779 p-values as graded evidence, but as evidence that can only speak against the null hypothesis,

780 would clearly help increasing inferential reproducibility by reducing the choices for qualitative

781 interpretation. For example, a large p-value would not be taken to support the null hypothesis,

782 and only rarely a result would be interpreted as providing no evidence at all.

783 As listed in the beginning of this paper, many authors have argued for removing fixed

784 thresholds. In 2016, Lemoine et al. (2016) wrote: "we join others in calling for a shift in the

785 statistical paradigm away from thresholds for statistical significance", and similar words were

786 used in the closing remarks of Greenland et al. (2016). So far, however, we see few researchers

787 performing this shift. Although black-and-white seems to give way to a more flexible approach

788 allowing for trends and marginal significance, dichotomous threshold thinking is still the rule

789 among applied researchers and even among many statisticians (McShane \& Gal 2016; McShane

$790 \&$ Gal 2017). Perhaps this is because of serious issues with interpreting p-values as graded

791 evidence?

792

793

\section{Arguments against removing thresholds}

794

795

In the following, we discuss potential problems. We start each paragraph with a possible

796

argument that could be raised against removing fixed significance thresholds.

797

798

'We need more stringent decision rules'

799

800 Evidently, removing significance thresholds "may lead to an increased prevalence of findings

801 that provide weak evidence, at best, against the null hypothesis" (Pritschet, Powell \& Horne

802 2016). This will not cause any problem, as long as the authors of a study acknowledge that they

803 found only weak evidence. Quite the contrary, publishing weak evidence is necessary to reduce

804 publication bias and truth inflation. However, p-values just below 0.05 are currently interpreted

805 as generally allowing a decision to reject the null hypothesis, which is one of the reasons why

806 scientific claims may often be unreliable (Oakes 1986; Sterne \& Smith 2001; Johnson 2013;

807 Colquhoun 2014). One alternative proposition to enhance replicability of research was therefore 
808 not to remove thresholds, but rather to apply more stringent thresholds (Johnson 2013; Ioannidis

809 2014; Academy of Medical Sciences 2015). For example, it may be said that you should "not

810 regard anything greater than $\mathrm{p}<0.001$ as a demonstration that you have discovered something"

811 (Colquhoun 2014).

812 We agree it is currently too "easy for researchers to find large and statistically significant

813 effects that could arise from noise alone" (Gelman \& Carlin 2014). Interpreting p-values as

814 graded evidence would mean to mistrust p-values around 0.05 , or smaller, depending on the

815 circumstances and the scientific discipline. To announce a discovery in particle physics, a p-

816 value as small as 0.0000003 may be needed (Johnson 2014). Also in other disciplines, we should

817 often judge our evidence as strong only if the p-value is much smaller than 0.05 : if we want to

818 demonstrate a surprising, counterintuitive effect; if we know that our null hypothesis has a high

819 prior probability (Bayarri et al. 2016); if our sample size is large (Anderson, Burnham \&

820 Thompson 2000; Pericchi, Pereira \& Perez 2014); or if postulating an effect that in reality is

821 negligible would have serious practical consequences.

822 However, even a p-value of 0.1 or larger may be interpreted as sufficiently strong evidence: if

823 we collected our data truly randomized and by minimizing bias and measurement error; if we

824 stuck to our pre-planned protocol for data analysis without trying multiple alternative analyses; if

825 our effect size is small; or if claiming only weak evidence for an effect that in reality is

826 practically important would have serious consequences (Gaudart et al. 2014).

827 A large effect with a large p-value could potentially have much more impact in the real world 828 than a small effect with a small p-value (Lemoine et al. 2016), although in the first case, the 829 evidence against the null hypothesis is weaker (Fig. 1B, E). And in exploratory studies screening 830 data for possible effects, results may be considered interesting even if their $p$-values are large 831 (Madden, Shah \& Esker 2015). Since p-values from exploratory research should be taken as 832 descriptive with little inferential content (Berry 2016), such studies are perhaps the clearest cases 833 in which there is no need for significance thresholds (Lew 2016). When exploring "what the data 834 say" (Lew 2016), it simply makes no sense to listen to them only if $\mathrm{p}<0.05$. We should, however, 835 clearly state that the research was exploratory (Simmons, Nelson \& Simonsohn 2011; Berry 836 2016). And we should keep in mind that if we recommend effects for further investigation 837 because their p-values are small, our effect sizes are likely inflated and will probably be smaller 838 in a follow-up study. 
Another argument against stringent thresholds like $p=0.001$ is that sample sizes in fields like

840

841

842

843

844

845

846

847

848

849

850

851

852

853

854

855

856

857

858

859

860

861

862

863

864

865

866

867

868

869

biomedicine or animal behavior are often bound to be small for practical and ethical reasons (Nakagawa 2004; Gaudart et al. 2014; Academy of Medical Sciences 2015). With small or moderate sample sizes, the chances that small but important effects will not become significant is very high, and this applies both to more stringent thresholds and to a possible scenario in which the conventional threshold of 0.05 were reinforced.

Because smaller p-values usually come with larger sample sizes, the question whether we should aim for smaller p-values boils down to whether we should conduct fewer but larger or more but smaller studies. Confidence and precision clearly increase with sample size (Button et al. 2013a). Based on simulations, however, IntHout, Ioannidis \& Borm (2016) recommend doing rather several studies with a moderate power of $30 \%$ to $50 \%$ than one larger study that would need a sample size four times to twice as large, respectively, to obtain $80 \%$ power. The reason is that every study will suffer from some sort of bias in the way it is conducted or reported, and that "in a series of studies, it is less likely that all studies will suffer from the same type of bias; consequently, their composite picture may be more informative than the result of a single large trial" (IntHout, Ioannidis \& Borm 2016).

And of course, to be of any use, all of those studies should be published. Cumulative evidence often builds up from several studies with larger p-values that only when combined show clear evidence against the null hypothesis (Greenland et al. 2016, p. 343). Very possibly, more stringent thresholds would lead to even more results being left unpublished, enhancing publication bias (Gaudart et al. 2014; Gelman \& Robert 2014). What we call winner's curse, truth inflation or inflated effect sizes will become even more severe with more stringent thresholds (Button et al. 2013b). And one reason why 'false-positives' are vastly more likely than we think, or as we prefer to say, why the evidence is often vastly overestimated, is that we often flexibly try different approaches to analysis (Simmons, Nelson \& Simonsohn 2011; Gelman \& Loken 2014). Exploiting those researcher degrees of freedom, and related phenomena termed multiplicity, data dredging, or p-hacking, would probably become more severe if obtaining significant results were harder due to more stringent thresholds.

We think that while aiming at making our published claims more reliable, requesting more stringent fixed thresholds would achieve quite the opposite. 
870 'Sample sizes will decrease'

871

872 Ideally, significance thresholds should force researchers to think about the sizes of their samples

873 - this is perhaps the only advantage thresholds could potentially offer. If significance is no

874 longer required, it could happen that researchers more often content themselves with smaller 875 sample sizes.

876 However, the argument could as well be reversed: fixed significance thresholds may lead to

877 unreasonably small sample sizes. We have had significance thresholds for decades, and average

878 power of studies was constantly low (Sedlmeier \& Gigerenzer 1989; Button et al. 2013b).

879 Researchers seem often to rely on rules of thumb by selecting sample sizes they think will yield

880 p-values just below 0.05 , although it is clear that p-values in this order of magnitude are hardly

881 replicable (Vankov, Bowers \& Munafo 2014). Oakes (1986, p. 85-86) argues that the major

882 reason for the abundance of low-power studies is the belief that "experimental findings suddenly

883 assume the mantle of reality at the arbitrary 0.05 level of significance" - if a result becomes true

884 once a threshold is crossed, there is simply no need to strive for larger sample sizes.

885 In their summary of 44 reviews on statistical power, Smaldino \& McElreath (2016) found not

886 only that average power to detect small effects was $24 \%$, but that there was no sign of increase

887 over six decades. The authors blame an academic environment that only rewards significant

888 findings, because in such a system, "an efficient way to succeed is to conduct low power studies.

889 Why? Such studies are cheap and can be farmed for significant results" (Smaldino \& McElreath

890 2016). Similarly, Higginson \& Munafo (2016) suggest that "researchers acting to maximize their

891 fitness should spend most of their effort seeking novel results and conduct small studies that

892 have only 10\%-40\% statistical power."

893 Quite often, significance testing appears like a sort of gambling. Even a study with minimized

894 investment into sample sizes will yield significant results, if only enough variables are measured

895 and the right buttons in the statistical software are pushed. Small sample sizes further have it that

896 significant effects will probably be inflated, so novel and surprising results are almost

897 guaranteed. And we may have become somewhat addicted to this game - it is satisfying to feed

898 data into the machine and find out whether the right variables have turned significant.

899 Indeed, "Fisher offered the idea of p-values as a means of protecting researchers from

900 declaring truth based on patterns in noise. In an ironic twist, p-values are now often manipulated 
901 to lend credence to noisy claims based on small samples" (Gelman \& Loken 2014). And the

902 manipulation can happen completely unintentionally, "without the researcher performing any

903 conscious procedure of fishing through the data" (Gelman \& Loken 2014), just as a side-effect of

904 how the game of significance testing is usually applied.

905 We should discourage significance farming by insisting that a p-value of 0.02 is not

906 automatically convincing, and that there is no need to throw away a study with $p=0.2$. We hope

907 that removing significance thresholds will allow researchers to take the risk and put more effort

908 into larger studies, without the fear that all the effort could be wasted if there were

909 nonsignificance in the end.

910

911 'We need objective decisions'

912

913 Graded evidence means no strict criterion to decide whether there is evidence or not, no

914 algorithm that makes the decision for us. Can graded evidence be objectively interpreted?

915 Fisher's honorable aim was to develop a "rigorous and objective test" (Fisher 1922, p. 314), and

916 Neyman and Pearson made the test even more rigorous by introducing automatic decisions

917 between two competing hypotheses.

918 Unfortunately, it seems like "the original Neyman-Pearson framework has no utility outside

919 quality control type applications" (Hurlbert \& Lombardi 2009). In most studies, we do not

920 formally specify alternative hypotheses, and we tend to use small sample sizes, so that beta

921 errors are usually both high and unknown (Fidler et al. 2004). This is one reason why our Fisher-

922 Neyman-Pearson hybrid tests offer only an "illusion of objectivity" (Berger \& Berry 1988;

923 Gigerenzer 1993). Another reason is that all statistical methods require subjective choices

924 (Gelman \& Hennig 2017). Prior to calculating p-values, we make all kinds of personal decisions:

925 in formulating our research question, in selecting the variables to be measured, in determining

926 the data sampling scheme, the statistical model, the test statistic, how to verify whether model

927 assumptions are met, how to handle outliers, how to transform the data, which software to use.

928 We do all of that and are used to justifying our choices; but interestingly, when it comes to

929 interpreting test results, "one can feel widespread anxiety surrounding the exercise of informed

930 personal judgment" (Gigerenzer 1993). 
931 For statistical tests, our a priori assumptions about the true model may be, for example, that

932 residuals are independent and identically distributed. Since "it is impossible logically to

933 distinguish between model assumptions and the prior distribution of the parameter", using prior

934 information is not a feature peculiar to Bayesian inference, but a necessity for all scientific

935 inference (Box 1980). As explained by Oakes (1986, p. 114), the statistical schools differ in the

936 manner in which they employ this prior information.

937 Pearson (1962) wrote about his work with Neyman: "We were certainly aware that inferences

938 must make use of prior information", thus "we left in our mathematical model a gap for the

939 exercise of a more intuitive process of personal judgment in such matters - to use our

940 terminology - as the choice of the most likely class of admissible hypotheses, the appropriate

941 significance level, the magnitude of worthwhile effects and the balance of utilities." But these

942 judgments had to be made before data collection, and "every detail of the design, including

943 responses to all possible surprises in the incoming data, must be planned in advance" (Berger \&

944 Berry 1988). To change the plan after data collection is to violate the model (Oakes 1986).

945 We agree this is the way to go in confirmatory studies that replicate procedures from earlier

946 research. Sticking to the rules is one reason why results from replication studies are usually more

947 reliable than from exploratory studies (Gelman \& Loken 2014; Higginson \& Munafo 2016);

948 another reason is that confirmatory studies may suffer less from publication bias than exploratory

949 studies (Open Science Collaboration 2015). We remind, however, that even in ideal confirmatory

950 studies, significance is not to be expected (see above), thus significance thresholds should not be

951 applied to judge replication success. And in exploratory studies, we see even less utility for the

952 original Neyman-Pearson framework and their predefined significance thresholds. Such studies

953 have all the rights to interrogate the data repeatedly and intensively (Lew 2016), as long as they

954 are acknowledged to be exploratory (Gelman \& Loken 2014; Berry 2016), and, of course, as

955 long as they report all results irrespective of their p-values.

956 We thus side with Ronald Fisher that the decision how to deal with the null hypothesis should

957 reside with the investigator rather than be taken for him in the Neyman-Pearson manner (Oakes

958 1986, p. 114). It should be remembered, however, that the decision whether there is an important

959 effect cannot be answered in a single study, and that it is often more interesting to discuss the

960 size of the effect than to speculate on its mere existence (e.g., Cumming 2012; Gelman 2013a).

961 In many cases, "research requires no firm decision: it contributes incrementally to an existing 
962 body of knowledge" (Sterne \& Smith 2001). Or in the words of Rozeboom (1960): "The primary

963 aim of a scientific experiment is not to precipitate decisions, but to make an appropriate

964 adjustment in the degree to which one accepts, or believes, the hypothesis or hypotheses being

965 tested."

966

967

'Null hypotheses should be acceptable'

968

969

As stated earlier, most null hypotheses of an effect of exactly zero are likely wrong at least to a

970

small degree. This claim seems to be widely accepted for correlational research (Edwards,

971 Lindman \& Savage 1963; Meehl 1967; Cohen 1990; Tukey 1991; Cohen 1994; Lecoutre \&

972

Poitevineau 2014; Stahel 2016), although its general applicability and philosophical background

973 are debated (Hagen 1997; Hagen 1998; Thompson 1998; Krueger 2001). However, the claim

974 may not apply to experimental studies (Meehl 1990). A null hypothesis of an effect of exactly

975 zero may be valid "for true experiments involving randomization (e.g., controlled clinical trials) or when any departure from pure chance is meaningful (as in laboratory experiments on

977 clairvoyance)" (Cohen 1994).

978 Under such circumstances, we would like to be able to show that the null hypothesis is true.

979 But how should this be done, if any p-value that we calculate can only speak against the null

980 hypothesis (Delong \& Lang 1992)? Interpreting p-values as graded evidence will mean that we

981 can never support the null hypothesis, and that alternative hypotheses may be "virtually

982 unkillable", buried at "a vast graveyard of undead theories" (Ferguson \& Heene 2012, although

983 the authors coined those words decrying publication bias against 'null' results and not the use of 984 graded evidence).

985 So "how to accept the null hypothesis gracefully" (Greenwald 1975)? The hard truth is that p986 values from classical null hypothesis tests are not made for this task - whether or not we use 987 them as graded evidence. As we discussed at length, also nonsignificant results cannot not 988 support the null hypothesis, and high power is of no help (Greenland 2012). There is only one 989 way to attach a number to the probability of a null hypothesis: "use a range, rather than a point, 990 null hypothesis", and "compute the posterior probability of the null (range) hypothesis"

991 (Greenwald 1975), or use similar Bayesian methods (Gallistel 2009; Morey \& Rouder 2011;

992 Dienes 2014). Alternative procedures in a frequentist framework include equivalence tests 
993 (Levine et al. 2008) or the careful interpretation of effect sizes and confidence intervals, to

994 determine whether an effect is so precisely small as to be practically unimportant (Fig. 1D, E;

995 Cumming 2014, p. 17; Simonsohn 2015).

996

997 'We need to get rid of p-values'

998

999 So p-values vary enormously from sample to sample even if there is a true effect. Unless they are

1000 very small, $p$-values therefore give only unreliable evidence against the null hypothesis

1001 (Cumming 2012, p. 152). For this and other reasons, p-values should probably be viewed as

1002 descriptive statistics, not as formal quantifications of evidence (Lavine 2014). To make it worse,

1003 we usually translate the observed evidence against the null hypothesis into evidence in favor of

1004 an alternative hypothesis, without even mentioning the null hypothesis ('females were twice as

1005 large as males, $\mathrm{p}<0.001^{\prime}$ ). However, knowing that the data are unlikely under the null hypothesis

1006 is of little use unless we consider whether or not they are also unlikely under the alternative

1007 hypothesis (Sellke, Bayarri \& Berger 2001; Barber \& Ogle 2014). Since very often we are not

1008 honestly interested in describing evidence against a hypothesis, null hypothesis testing is usually

1009 a "parody of falsificationism in which straw-man null hypothesis A is rejected and this is taken

1010 as evidence in favor of preferred alternative B" (Gelman 2016).

1011 Why do we not join others and discourage using p-values and null hypothesis tests (e.g.,

1012 Carver 1978; Schmidt 1996; Ziliak \& McCloskey 2008; Orlitzky 2012; Cumming 2014;

1013 Trafimow \& Marks 2015; Gorard 2016)?

1014 We agree to (Cumming 2012, p. 33) that "thinking of $p$ as strength of evidence may be the

1015 least bad approach". We thus propose to describe our observed gradual evidence against the null

1016 hypothesis rather than to 'reject' the null. A first step would be to stop using the word 'significant'

1017 (Higgs 2013; Colquhoun 2014). Indeed, often null hypotheses about zero effects are

1018 automatically chosen only to be rejected (Gelman 2013b), and null hypotheses on effect sizes

1019 other than zero, or on ranges of effect sizes, would be more appropriate (Cohen 1994; Greenland

1020 et al. 2016). The way to become aware of our zero effect automatism is to "replace the word

1021 significant with a concise and defensible description of what we actually mean to evoke by using 1022 it" (Higgs 2013). 
1023 These are small changes that may look insufficient to some. But the beauty in such simple 1024 measures to help address the crisis of unreplicable research is that they do not hurt. We do not 1025 need to publicly register our study protocol before we collect data, we do not need to learn new 1026 statistics, we do not even need to use confidence intervals if we prefer to use standard errors. Of 1027 course all of those measures would be helpful (e.g., Cumming 2014; Academy of Medical 1028 Sciences 2015), but they usually require an active change of research practices. And many 1029 researchers seem to hesitate changing statistical procedures that have been standard for nearly a 1030 century (Thompson 1999; Sharpe 2013).

1031 Although there are hundreds of papers arguing against null hypothesis significance testing, we 1032 see more and more p-values (Chavalarias et al. 2016) and the ASA feeling obliged to tell us how 1033 to use them properly (Goodman 2016; Wasserstein \& Lazar 2016). Apparently, bashing or 1034 banning p-values does not work. We need a smaller incremental step that at the same time is 1035 highly efficient. There are not many easy ways to improve scientific inference, but together with 1036 Higgs (2013) and others, we believe that removing significance thresholds is one of them.

Also, we do not deny that some thresholds are necessary when interpreting statistics. If we

1038 want to draw error bars around a mean, the lines of the bars must end at some point that is 1039 defined by a threshold. By convention, we use 95\% confidence intervals, cutting off parameter 1040 values that would be rejected at the $\mathrm{p}=0.05$ threshold; of course we could also use $90 \%, 80 \%$ or $104175 \%$ confidence intervals with $0.10,0.20$ or 0.25 thresholds (Hurlbert \& Lombardi 2009). Geoff

1042 Cumming's (2012, p. 76) recommendation is to concentrate on 95\% confidence intervals, 1043 because "it's challenging enough to build up good intuitions about the standard 95\% level of 1044 confidence".

1045 Unfortunately, many researchers seem to use confidence intervals mostly to decide whether 1046 the null value is excluded, thus converting them to significance tests (Hoekstra, Johnson \& Kiers 1047 2012; McCormack, Vandermeer \& Allan 2013; Rothman 2014; Savalei \& Dunn 2015; van 1048 Helden 2016). Potentially, using confidence intervals could encourage estimation thinking and 1049 meta-analytic thinking (Cumming 2014), but for full benefit, researchers would need to interpret 1050 confidence intervals without recourse to significance (Coulson et al. 2010). 'Cat's-eye' pictures of $105195 \%$ confidence intervals show how the plausibility that a value is the true population average is 1052 greatest for values near our point estimate, in the center of the interval; plausibility then drops 1053 smoothly to either end of the confidence interval, then continues to drop further outside the 
1054 interval (Cumming 2012, p. 95). This means that "we should not lapse back into dichotomous

1055 thinking by attaching any particular importance to whether a value of interest lies just inside or 1056 just outside our confidence interval" (Cumming 2014).

1057 Further, we recommend choosing not only from the available null hypothesis tests but also 1058 from the toolbox provided by Bayesian statistics (Korner-Nievergelt et al. 2015). But we agree 1059 that we should not "look for a magic alternative to NHST [null hypothesis significance testing], 1060 some other objective mechanical ritual to replace it. It doesn't exist" (Cohen 1994).

1061 Whatever method of statistical inference we use, biased effect sizes will be a problem with 1062 criteria that are used to select results for publication or interpretation. If effect sizes are reported 1063 because they are large, this will of course create an upwards bias, similarly to selecting p-values

1064 because they are small. If effect sizes are reported because they are small, or p-values because 1065 they are large, for example when people are interested to show that some treatment may not have 1066 an (adverse) effect, this will create a downwards bias (Greenland et al. 2016). If results are

1067 reported because Bayesian posterior probabilities are at least three times larger for an alternative 1068 than for a null hypothesis, this is equivalent to selective reporting based on significance 1069 thresholds (Simonsohn 2014).

1070 Inflated effects will occur when a discovery is claimed because a Bayes factor is better than a 1071 given value or a false discovery rate is below a given value (Button et al. 2013b). Selecting a

1072 model because the difference in the Akaike information criterion ( $\triangle \mathrm{AIC}$ ) passes some threshold

1073 is equivalent to model selection based on significance and will generate inflated effects

1074 (Murtaugh 2014a; Murtaugh 2014b; Parker et al. 2016). Whenever we use effect sizes,

1075 confidence intervals, AIC, posteriors, Bayes factors, likelihood ratios, or false discovery rates in

1076 threshold tests and decision heuristics for reporting or interpreting selected results rather than all

1077 results, we create biases and invalidate the answers we give to our questions (Simonsohn 2014;

1078 Yu et al. 2014; Parker et al. 2016; Wasserstein \& Lazar 2016).

1079 However, the greatest source of bias probably comes from selectively reporting small p-

1080 values, simply because of the dominant role of null hypothesis significance testing. If we learn to

1081 judge the strength of evidence based not on the event of passing a threshold, but on graded

1082 summaries of data like the p-value, we will become more aware of uncertainty. And statistical

1083 methods are not simply applied to a discipline but change the discipline itself (Gigerenzer \&

1084 Marewski 2015). A greater acceptance of uncertainty and embracing of variation (Gelman 2016) 
1085 could shift our focus back to core values like discussing practical importance of effect sizes,

1086 minimizing measurement error, performing replication studies, and using informed personal

1087 judgment (Cumming 2014; Gigerenzer \& Marewski 2015; Lemoine et al. 2016).

1088

1089 Conclusions

1090

1091 Part of an apparent crisis of unreplicable research is caused by the way we use statistics for

1092 analyzing, interpreting and communicating our data. Applying significance thresholds leads to

1093 overconfidence in small but highly variable p-values, to publication bias against larger p-values,

1094 and to reported effects that are biased upwards. But larger p-values are to be expected also if

1095 there is a true effect, and they must be published because otherwise smaller p-values are

1096 uninterpretable. Thus, smaller p-values need to lose reputation, and larger p-values need to gain

1097 reputation. This is best accomplished by removing fixed significance thresholds, by cautiously

1098 interpreting p-values as graded evidence against the null hypothesis, and by putting more

1099 emphasis on interpreting effect sizes and interval estimates, using non-automated informed

1100 judgment. We give the last word to Edwin G. Boring (1919), who one century ago wrote in his

1101 paper "Mathematical vs. scientific significance": "Conclusions must ultimately be left to the

1102 scientific intuition of the experimenter and his public."

1103

\section{Acknowledgements}

1105

1106 For helpful comments on the manuscript we thank Daniel Berner, Lilla Lovász, the students and 1107 colleagues from our journal clubs, and the three referees.

\section{References}

Academy of Medical Sciences 2015. Reproducibility and reliability of biomedical research: improving research practice. Symposium report. Academy of Medical Sciences, BBSRC,

1113 MRC, Wellcome Trust. 
1114 Anderson DR, Burnham KP, Thompson WL. 2000. Null hypothesis testing: Problems,

1115 prevalence, and an alternative. Journal of Wildlife Management 64:912-923.

$1116 \quad 10.2307 / 3803199$

1117 Badenes-Ribera L, Frias-Navarro D, Iotti B, Bonilla-Campos A, Longobardi C. 2016.

1118 Misconceptions of the p-value among Chilean and Italian academic psychologists.

$1119 \quad$ Frontiers in Psychology 7:1247. 10.3389/fpsyg.2016.01247

1120 Baker M. 2016. Is there a reproducibility crisis? Nature 533:452-454.

1121 Barber JJ, Ogle K. 2014. To P or not to P? Ecology 95:621-626. 10.1890/13-1402.1

1122 Bayarri MJ, Benjamin DJ, Berger JO, Sellke TM. 2016. Rejection odds and rejection ratios: A

1123 proposal for statistical practice in testing hypotheses. Journal of Mathematical

1124 Psychology 72:90-103. 10.1016/j.jmp.2015.12.007

1125 Berger JO, Berry DA. 1988. Statistical analysis and the illusion of objectivity. American

1126 Scientist 76:159-165.

1127 Berkson J. 1938. Some difficulties of interpretation encountered in the application of the chi-

1128 square test. Journal of the American Statistical Association 33:526-536.

$1129 \quad 10.2307 / 2279690$

1130 Berry DA. 2016. P-values are not what theyre cracked up to be. The American Statistician,

1131 supplemental material to the ASA statement on p-values and statistical significance.

$1132 \quad 10.1080 / 00031305.2016 .1154108$

1133 Bishop DVM, Thompson PA. 2016. Problems in using p-curve analysis and text-mining to detect

1134 rate of p-hacking and evidential value. PeerJ 4:e1715. 10.7717/peerj.1715

1135 Boos DD, Stefanski LA. 2011. P-value precision and reproducibility. American Statistician

$1136 \quad 65: 213-221.10 .1198 / \operatorname{tas} .2011 .10129$

1137 Boring EG. 1919. Mathematical vs. scientific significance. Psychological Bulletin 16:335-338.

$1138 \quad 10.1037 / \mathrm{h} 0074554$

1139 Box GEP. 1980. Sampling and Bayes inference in scientific modeling and robustness. Journal of $1140 \quad$ the Royal Statistical Society, Series A 143:383-430. 10.2307/2982063

1141 Branch M. 2014. Malignant side effects of null-hypothesis significance testing. Theory \&

$1142 \quad$ Psychology 24:256-277. 10.1177/0959354314525282

1143 Brembs B, Button K, Munafo M. 2013. Deep impact: unintended consequences of journal rank.

1144 Frontiers in Human Neuroscience 7:291. 10.3389/fnhum.2013.00291 
1145 Button KS, Ioannidis JPA, Mokrysz C, Nosek BA, Flint J, Robinson ESJ, Munafo MR. $2013 a$.

1146

1147

1148

1149

1150

1151

1152

1153

1154

1155

1156

1157

1158

1159

1160

1161

1162

1163

1164

1165

1166

1167

1168

1169

1170

1171

1172

1173

1174

Confidence and precision increase with high statistical power. Nature Reviews

Neuroscience 14. 10.1038/nrn3475-c4

Button KS, Ioannidis JPA, Mokrysz C, Nosek BA, Flint J, Robinson ESJ, Munafo MR. 2013 b.

Power failure: why small sample size undermines the reliability of neuroscience. Nature Reviews Neuroscience 14:365-376. 10.1038/nrn3475

Campbell JP. 1982. Editorial: Some remarks from the outgoing editor. Journal of Applied Psychology 67:691-700.

Capen EC, Clapp RV, Campbell WM. 1971. Competitive bidding in high-risk situations. Journal of Petroleum Technology 23:641-653.

Carver RP. 1978. Case against statistical significance testing. Harvard Educational Review 48:378-399.

Chavalarias D, Wallach JD, Li AH, Ioannidis JP. 2016. Evolution of reporting P values in the biomedical literature, 1990-2015. JAMA-Journal of the American Medical Association 315:1141-1148. 10.1001/jama.2016.1952

Cohen J. 1962. The statistical power of abnormal-social psychological research: A review. Journal of Abnormal Psychology 65:145-153. 10.1037/h0045186

Cohen J. 1990. Things I have learned (so far). American Psychologist 45:1304-1312. 10.1037//0003-066x.45.12.1304

Cohen J. 1994. The earth is round $(\mathrm{p}<.05)$. American Psychologist 49:997-1003. 10.1037/0003066x.50.12.1103

Colquhoun D. 2014. An investigation of the false discovery rate and the misinterpretation of pvalues. Royal Society Open Science 1:140216. 10.1098/rsos.140216

Coulson M, Healey M, Fidler F, Cumming G. 2010. Confidence intervals permit, but do not guarantee, better inference than statistical significance testing. Frontiers in Psychology $1: 26.10 .3389 /$ fpsyg.2010.00026

Cowles M, Davis C. 1982a. Is the .05 level subjectively reasonable? Canadian Journal of Behavioural Science 14:248-252. 10.1037/h0081256

Cowles M, Davis C. 1982b. On the origins of the .05 level of statistical significance. American Psychologist 37:553-558. 10.1037/0003-066x.37.5.553 
1175 Csada RD, James PC, Espie RHM. 1996. The "file drawer problem" of non-significant results:

1176 Does it apply to biological research? Oikos 76:591-593. 10.2307/3546355

1177 Cumming G. 2007. Inference by eye: Pictures of confidence intervals and thinking about levels 1178 of confidence. Teaching Statistics 29:89-93.

1179 Cumming G. 2008. Replication and p intervals. Perspectives on Psychological Science 3:286$1180 \quad 300.10 .1111 / j .1745-6924.2008 .00079 . x$

1181 Cumming G. 2012. Understanding the new statistics. New York: Routledge.

1182 Cumming G. 2014. The new statistics: why and how. Psychological Science 25:7-29.

$1183 \quad 10.1177 / 0956797613504966$

1184 de Winter JCF, Dodou D. 2015. A surge of p-values between 0.041 and 0.049 in recent decades

1185 (but negative results are increasing rapidly too). PeerJ 3:e733. 10.7717/peerj.733

1186 Delong JB, Lang K. 1992. Are all economic hypotheses false? Journal of Political Economy

$1187 \quad 100: 1257-1272$.

1188 Dienes Z. 2014. Using Bayes to get the most out of non-significant results. Frontiers in

$1189 \quad$ Psychology 5:781. 10.3389/fpsyg.2014.00781

1190 Dixon P. 2003. The p-value fallacy and how to avoid it. Canadian Journal of Experimental $1191 \quad$ Psychology 57:189-202. 10.1037/h0087425

1192 Drummond GB. 2016. Most of the time, P is an unreliable marker, so we need no exact cut-off.

1193 British Journal of Anaesthesia 116:893-893. 10.1093/bja/aew146

1194 Dwan K, Gamble C, Williamson PR, Kirkham JJ, the Reporting Bias Group. 2013. Systematic

1195 review of the empirical evidence of study publication bias and outcome reporting bias -

$1196 \quad$ an updated review. PLoS One 8:e66844. 10.1371/journal.pone.0066844

1197 Edgington ES. 1970. Hypothesis testing without fixed levels of significance. Journal of $1198 \quad$ Psychology 76:109-115.

1199 Edwards W, Lindman H, Savage LJ. 1963. Bayesian statistical inference for psychological $1200 \quad$ research. Psychological Review 70:193-242. 10.1037/h0044139

1201 Eysenck HJ. 1960. The concept of statistical significance and the controversy about one-tailed tests. Psychological Review 67:269-271. 10.1037/h0048412

1203 Fabrigar LR, Wegener DT. 2016. Conceptualizing and evaluating the replication of research results. Journal of Experimental Social Psychology 66:68-80. 10.1016/j.jesp.2015.07.009 
1205 Fanelli D. 2012. Negative results are disappearing from most disciplines and countries. Scientometrics 90:891-904. 10.1007/s11192-011-0494-7

1207 Fanelli D, Costas R, Ioannidis JPA. 2017. Meta-assessment of bias in science. Proceedings of the National Academy of Sciences of the United States of America 114:3714-3719. 10.1073/pnas.1618569114

Ferguson CJ, Heene M. 2012. A vast graveyard of undead theories: publication bias and psychological science's aversion to the null. Perspectives on Psychological Science 7:555-561. 10.1177/1745691612459059

1213 Fidler F, Burgman MA, Cumming G, Buttrose R, Thomason N. 2006. Impact of criticism of null-hypothesis significance testing on statistical reporting practices in conservation biology. Conservation Biology 20:1539-1544. 10.1111/j.1523-1739.2006.00525.x

Fidler F, Geoff C, Mark B, Neil T. 2004. Statistical reform in medicine, psychology and ecology. The Journal of Socio-Economics 33:615-630. 10.1016/j.socec.2004.09.035

Finch S, Cumming G, Thomason N. 2001. Reporting of statistical inference in the Journal of Applied Psychology: Little evidence of reform. Educational and Psychological Measurement 61:181-210. 10.1177/00131640121971167

Fisher RA. 1922. On the mathematical foundations of theoretical statistics. Philosophical Transactions of the Royal Society of London, Series A 222:309-368.

Fisher RA. 1925. Statistical methods for research workers. Edinburgh: Oliver and Boyd.

Fisher RA. 1926. The arrangement of field experiments. Journal of the Ministry of Agriculture of Great Britain 33:503-513.

Fisher RA. 1929. The statistical method in psychical research. Proceedings of the Society for Psychical Research 39:189-192.

1228 Fisher RA. 1935. Statistical tests. Nature 136:474-474.

1229 Fisher RA. 1937. The design of experiments. 2nd edition. Edinburgh: Oliver and Boyd.

1230 Fisher R. 1955. Statistical methods and scientific induction. Journal of the Royal Statistical $1231 \quad$ Society Series B-Statistical Methodology 17:69-78.

1232 Fisher RA. 1956. Statistical methods and scientific inference. Edinburgh: Oliver and Boyd.

1233 Fisher RA. 1958. Statistical methods for research workers. 13th edition. Edinburgh: Oliver and 1234 Boyd. 
1235 Foreman P, Murnighan JK. 1996. Learning to avoid the winner's curse. Organizational Behavior

1236

1237

1238

1239

1240

1241

1242

1243

1244

1245

1246

1247

1248

1249

1250

1251

1252

1253

1254

1255

1256

1257

1258

1259

1260

1261

1262

1263

1264 and Human Decision Processes 67:170-180. 10.1006/obhd.1996.0072

Forstmeier W, Schielzeth H. 2011. Cryptic multiple hypotheses testing in linear models: overestimated effect sizes and the winner's curse. Behavioral Ecology and Sociobiology 65:47-55. 10.1007/s00265-010-1038-5

Francis G. 2013. Replication, statistical consistency, and publication bias. Journal of Mathematical Psychology 57:153-169. 10.1016/j.jmp.2013.02.003

Gallistel CR. 2009. The importance of proving the null. Psychological Review 116:439-453. $10.1037 / \mathrm{a} 0015251$

Garner C. 2007. Upward bias in odds ratio estimates from genome-wide association studies. Genetic Epidemiology 31:288-295. 10.1002/gepi.20209

Gaudart J, Huiart L, Milligan PJ, Thiebaut R, Giorgi R. 2014. Reproducibility issues in science, is $\mathrm{P}$ value really the only answer? Proceedings of the National Academy of Sciences of the United States of America 111:E1934-E1934. 10.1073/pnas.1323051111

Gelman A. 2013a. Interrogating p-values. Journal of Mathematical Psychology 57:188-189. 10.1016/j.jmp.2013.03.005

Gelman A. 2013b. The problem with p-values is how they're used. Available at http://www.stat.columbia.edu/ gelman (accessed 6 June 2017)

Gelman A. 2015. The connection between varying treatment effects and the crisis of unreplicable research: A Bayesian perspective. Journal of Management 41:632-643. $10.1177 / 0149206314525208$

Gelman A. 2016. The problems with p-values are not just with p-values. The American Statistician, supplemental material to the ASA statement on p-values and statistical significance. 10.1080/00031305.2016.1154108

Gelman A, Carlin J. 2014. Beyond power calculations: assessing type S (sign) and type M (magnitude) errors. Perspectives on Psychological Science 9:641-651. $10.1177 / 1745691614551642$

Gelman A, Hennig C. 2017. Beyond subjective and objective in statistics. Journal of the Royal Statistical Society, Series A (Statistics in Society) 180.

Gelman A, Loken E. 2014. The statistical crisis in science. American Scientist 102:460-465. 
1265 Gelman A, Robert CP. 2014. Revised evidence for statistical standards. Proceedings of the

1266

1267

1268

1269

1270

1271

1272

1273

1274

1275

1276

1277

1278

1279

1280

1281

1282

1283

1284

1285

1286

1287

1288

1289

1290

1291

1292

1293

1294

1295 National Academy of Sciences of the United States of America 111:E1933-E1933. 10.1073/pnas.1322995111

Gelman A, Stern H. 2006. The difference between "significant" and "not significant" is not itself statistically significant. American Statistician 60:328-331. 10.1198/000313006x152649

Gerber AS, Malhotra N. 2008. Publication bias in empirical sociological research - Do arbitrary significance levels distort published results? Sociological Methods \& Research 37:3-30. $10.1177 / 0049124108318973$

Gigerenzer G. 1993. The superego, the ego, and the id in statistical reasoning. In: Hillsdale NJ, ed. A handbook for data analysis in the behavioral sciences. Hillsdale: Lawrence Erlbaum Associates, 311-339.

Gigerenzer G, Krauss S, Vitouch O. 2004. The null ritual: What you always wanted to know about significance testing but were afraid to ask. In: Kaplan D, ed. The Sage handbook of quantitative methodology for the social sciences. Thousand Oaks: Sage Publications, 391-408.

Gigerenzer G, Marewski JN. 2015. Surrogate science: The idol of a universal method for scientific inference. Journal of Management 41:421-440. 10.1177/0149206314547522

Gigerenzer G, Swijtink Z, Porter T, Daston L, Beatty J, Krüger L. 1989. The empire of chance: How probability changed science and everyday life. New York: Cambridge University Press.

Gill J. 1999. The insignificance of null hypothesis significance testing. Political Research Quarterly 52:647-674. 10.2307/449153

Giner-Sorolla R. 2016. Approaching a fair deal for significance and other concerns. Journal of Experimental Social Psychology 65:1-6. 10.1016/j.cjesp.2016.01.010

Goodman SN. 1992. A comment on replication, p-values and evidence. Statistics in Medicine 11:875-879. 10.1002/sim.4780110705

Goodman SN. 1993. P values, hypothesis tests, and likelihood: Implications for epidemiology of a neglected historical debate. American Journal of Epidemiology 137:485-496.

Goodman SN. 2016. The next questions: who, what, when, where, and why? The American Statistician, supplemental material to the ASA statement on p-values and statistical significance. 10.1080/00031305.2016.1154108 
1296 Goodman SN, Fanelli D, Ioannidis JPA. 2016. What does research reproducibility mean?

$1297 \quad$ Science Translational Medicine 8. 10.1126/scitranslmed.aaf5027

1298 Gorard S. 2016. Damaging real lives through obstinacy: re-emphasising why significance testing 1299 is wrong. Sociological Research Online 21. 10.5153/sro.3857

1300 Göring HHH, Terwilliger JD, Blangero J. 2001. Large upward bias in estimation of locusspecific effects from genomewide scans. American Journal of Human Genetics 69:13571369. $10.1086 / 324471$

Greenland S. 2012. Nonsignificance plus high power does not imply support for the null over the alternative. Annals of Epidemiology 22:364-368. 10.1016/j.annepidem.2012.02.007

Greenland S, Poole C. 2013. Living with statistics in observational research. Epidemiology 24:73-78. 10.1097/EDE.0b013e3182785a49

Greenland S, Senn SJ, Rothman KJ, Carlin JB, Poole C, Goodman SN, Altman DG. 2016. Statistical tests, $\mathrm{P}$ values, confidence intervals, and power: a guide to misinterpretations. European Journal of Epidemiology 31:337-350. 10.1007/s10654-016-0149-3

Greenwald AG. 1975. Consequences of prejudice against the null hypothesis. Psychological Bulletin 82:1-19. 10.1037/h0076157

Greenwald AG, Gonzalez R, Harris RJ, Guthrie D. 1996. Effect sizes and p values: What should be reported and what should be replicated? Psychophysiology 33:175-183. 10.1111/j.1469-8986.1996.tb02121.x

Hagen RL. 1997. In praise of the null hypothesis statistical test. American Psychologist 52:1524. 10.1037/0003-066x.52.1.15

Hagen RL. 1998. A further look at wrong reasons to abandon statistical testing. American Psychologist 53:801-803. 10.1037/0003-066x.53.7.801

Halsey LG, Curran-Everett D, Vowler SL, Drummond GB. 2015. The fickle P value generates irreproducible results. Nature Methods 12:179-185. 10.1038/nmeth.3288

Higginson AD, Munafo MR. 2016. Current incentives for scientists lead to underpowered studies with erroneous conclusions. PLoS Biology 14:e2000995. 10.1371/journal.pbio.2000995

1323 Higgs MD. 2013. Do we really need the s-word? American Scientist 101:6-9.

1324 Hoekstra R, Finch S, Kiers HAL, Johnson A. 2006. Probability as certainty: Dichotomous thinking and the misuse of $\mathrm{p}$ values. Psychonomic Bulletin \& Review 13:1033-1037. 
1327 Hoekstra R, Johnson A, Kiers HAL. 2012. Confidence intervals make a difference: Effects of 1328 showing confidence intervals on inferential reasoning. Educational and Psychological Measurement 72:1039-1052. 10.1177/0013164412450297

Hubbard R, Bayarri MJ. 2003. Confusion over measures of evidence (p's) versus errors ( $\alpha$ 's) in classical statistical testing. American Statistician 57:171-178. 10.1198/0003130031856

Hung HMJ, Oneill RT, Bauer P, Kohne K. 1997. The behavior of the p-value when the alternative hypothesis is true. Biometrics 53:11-22. 10.2307/2533093

Hurlbert SH, Lombardi CM. 2009. Final collapse of the Neyman-Pearson decision theoretic framework and rise of the neoFisherian. Annales Zoologici Fennici 46:311-349.

IntHout J, Ioannidis JPA, Borm GF. 2016. Obtaining evidence by a single well-powered trial or several modestly powered trials. Statistical Methods in Medical Research 25:538-552. $10.1177 / 0962280212461098$

Ioannidis JPA. 2005. Contradicted and initially stronger effects in highly cited clinical research. JAMA-Journal of the American Medical Association 294:218-228. 10.1001/jama.294.2.218

Ioannidis JPA. 2008. Why most discovered true associations are inflated. Epidemiology 19:640648. 10.1097/EDE.0b013e31818131e7

Ioannidis JPA. 2010. Meta-research: The art of getting it wrong. Research Synthesis Methods 1:169-184. 10.1002/jrsm.19

Ioannidis JPA. 2014. How to make more published research true. PLoS Medicine 11:e1001747. 10.1371/journal.pmed.1001747

Ioannidis JPA, Greenland S, Hlatky MA, Khoury MJ, Macleod MR, Moher D, Schulz KF, Tibshirani R. 2014. Increasing value and reducing waste in research design, conduct, and analysis. Lancet 383:166-175. 10.1016/s0140-6736(13)62227-8

Jennions MD, Møller AP. 2002. Relationships fade with time: a meta-analysis of temporal trends

1354 Jennions MD, Møller AP. 2003. A survey of the statistical power of research in behavioral ecology and animal behavior. Behavioral Ecology 14:438-445. 10.1093/beheco/14.3.438 
1356 John LK, Loewenstein G, Prelec D. 2012. Measuring the prevalence of questionable research

1357

1358

1359

1360

1361

1362

1363

1364

1365

1366

1367

1368

1369

1370

1371

1372

1373

1374

1375

1376

1377

1378

1379

1380

1381

1382

1383

1384

1385 practices with incentives for truth telling. Psychological Science 23:524-532. $10.1177 / 0956797611430953$

Johnson DH. 1999. The insignificance of statistical significance testing. Journal of Wildlife Management 63:763-772. 10.2307/3802789

Johnson VE. 2013. Revised standards for statistical evidence. Proceedings of the National Academy of Sciences of the United States of America 110:19313-19317. 10.1073/pnas. 1313476110

Johnson VE. 2014. Reply to Gelman, Gaudart, Pericchi: More reasons to revise standards for statistical evidence. Proceedings of the National Academy of Sciences of the United States of America 111:E1936-E1937. 10.1073/pnas.1400338111

Kelly CD. 2006. Replicating empirical research in behavioral ecology: How and why it should be done but rarely ever is. Quarterly Review of Biology 81:221-236. 10.1086/506236

Kline RB. 2013. Beyond significance testing: Statistics reform in the behavioral sciences. Washington: American Psychological Association.

Korner-Nievergelt F, Hüppop O. 2016. Kurze Einführung in Bayes-Statistik mit R für Ornithologen. Vogelwarte 54:181-194.

Korner-Nievergelt F, Roth T, von Felten S, Guélat J, Almasi B, Korner-Nievergelt P. 2015. Bayesian data analysis in ecology using linear models with R, BUGS, and Stan. London: Academic Press.

Krueger J. 2001. Null hypothesis significance testing - On the survival of a flawed method. American Psychologist 56:16-26. 10.1037//0003-066x.56.1.16

Labovitz S. 1968. Criteria for selecting a significance level: A note on the sacredness of .05. American Sociologist 3:220-222.

Lai J, Fidler F, Cumming G. 2012. Subjective p intervals - Researchers underestimate the variability of $\mathrm{p}$ values over replication. Methodology 8:51-62. 10.1027/1614$2241 / \mathrm{a} 000037$

Lavine M. 2014. Comment on Murtaugh. Ecology 95:642-645. 10.1890/13-1112.1

Lazzeroni LC, Lu Y, Belitskaya-Levy I. 2014. P-values in genomics: Apparent precision masks high uncertainty. Molecular Psychiatry 19:1336-1340. 10.1038/mp.2013.184 
1386 Lecoutre B, Poitevineau J. 2014. The significance test controversy revisited. Heidelberg: 1387 Springer.

1388 Lecoutre M-P, Poitevineau J, Lecoutre B. 2003. Even statisticians are not immune to 1389 misinterpretations of Null Hypothesis Significance Tests. International Journal of $1390 \quad$ Psychology 38:37-45. 10.1080/00207590244000250

1391 Leek JT, Jager LR. 2017. Is most published research really false? Annual Review of Statistics 1392 and Its Application 4:109-122. 10.1146/annurev-statistics-060116-054104

1393 Lehmann EL. 2011. Fisher, Neyman, and the creation of classical statistics. New York: $1394 \quad$ Springer.

1395 Lemoine NP, Hoffman A, Felton AJ, Baur L, Chaves F, Gray J, Yu Q, Smith MD. 2016. Underappreciated problems of low replication in ecological field studies. Ecology 97:2554-2561. 10.1002/ecy.1506

Lenhard J. 2006. Models and statistical inference: The controversy between Fisher and NeymanPearson. British Journal for the Philosophy of Science 57:69-91. 10.1093/bjps/axi152 Lertzman KP. 1995. Notes on writing papers and theses. Bulletin of the Ecological Society of America 76:86-90.

Levine TR, Weber R, Park HS, Hullett CR. 2008. A communication researchers' guide to null hypothesis significance testing and alternatives. Human Communication Research 34:188-209. 10.1111/j.1468-2958.2008.00318.x

Lew MJ. 2016. Three inferential questions, two types of p-value. The American Statistician, supplemental material to the ASA statement on p-values and statistical significance. 10.1080/00031305.2016.1154108

Lisse JR, Perlman M, Johansson G, Shoemaker JR, Schechtman J, Skalky CS, Dixon ME, Polls

1415 Lovasich JL, Neyman J, Scott EL, Wells MA. 1971. Hypothetical explanations of negative AB, Mollen AJ, Geba GP. 2003. Gastrointestinal tolerability and effectiveness of rofecoxib versus naproxen in the treatment of osteoarthritis - A randomized, controlled trial. Annals of Internal Medicine 139:539-546.

Loftus GR. 1993. A picture is worth 1000 p-values: On the irrelevance of hypothesis-testing in the microcomputer age. Behavior Research Methods Instruments \& Computers 25:250256. $10.3758 / \mathrm{bf} 03204506$ apparent effects of cloud seeding in whitetop experiment. Proceedings of the National 
Madden LV, Shah DA, Esker PD. 2015. Does the P value have a future in plant pathology? Phytopathology 105:1400-1407. 10.1094/phyto-07-15-0165-le

Maxwell SE, Lau MY, Howard GS. 2015. Is psychology suffering from a replication crisis? What does "failure to replicate" really mean? American Psychologist 70:487-498. 10.1037/a0039400

McCarthy MA. 2007. Bayesian methods for ecology. Cambridge: Cambridge University Press. McCormack J, Vandermeer B, Allan GM. 2013. How confidence intervals become confusion intervals. BMC Medical Research Methodology 13:134. 10.1186/1471-2288-13-134

McShane BB, Gal D. 2016. Blinding us to the obvious? The effect of statistical training on the evaluation of evidence. Management Science 62:1707-1718. 10.1287/mnsc.2015.2212

McShane BB, Gal D. 2017. Statistical significance and the dichotomization of evidence: The relevance of the ASA statement on statistical significance and p-values for statisticians. Journal of the American Statistical Association 112.

Meehl PE. 1967. Theory-testing in psychology and physics: A methodological paradox. Philosophy of Science 34:103-115. 10.1086/288135

Meehl PE. 1990. Why summaries of research on psychological theories are often uninterpretable. Psychological Reports 66:195-244. 10.2466/pr0.66.1.195-244

Miller J. 2009. What is the probability of replicating a statistically significant effect? Psychonomic Bulletin \& Review 16:617-640. 10.3758/pbr.16.4.617

Morey RD, Rouder JN. 2011. Bayes factor approaches for testing interval null hypotheses. Psychological Methods 16:406-419. 10.1037/a0024377

Munafo MR, Flint J. 2010. How reliable are scientific studies? British Journal of Psychiatry 197:257-258. 10.1192/bjp.bp.109.069849

1444 Mundry R. 2011. Issues in information theory-based statistical inference - A commentary from a

Munafo MR, Stothart G, Flint J. 2009. Bias in genetic association studies and impact factor. Molecular Psychiatry 14:119-120. 10.1038/mp.2008.77 
1447 Murdoch DJ, Tsai Y-L, Adcock J. 2008. P-values are random variables. American Statistician 62:242-245. 10.1198/000313008x332421

1449

Murtaugh PA. 2014a. In defense of P values. Ecology 95:611-617. 10.1890/13-0590.1

Murtaugh PA. 2014b. Rejoinder. Ecology 95:651-653. 10.1890/13-1858.1

Nakagawa S. 2004. A farewell to Bonferroni: The problems of low statistical power and publication bias. Behavioral Ecology 15:1044-1045. 10.1093/beheco/arh107

Neyman J. 1977. Frequentist probability and frequentist statistics. Synthese 36:97-131. $10.1007 / \mathrm{bf00485695}$

Neyman J, Pearson ES. 1933a. On the problem of the most efficient tests of statistical hypotheses. Philosophical Transactions of the Royal Society of London, Series A 231:289-337. 10.1098/rsta.1933.0009

Neyman J, Pearson ES. 1933b. The testing of statistical hypotheses in relation to probabilities a priori. Proceedings of the Cambridge Philosophical Society 29:492-510.

Nosek BA, Errington TM. 2017. Making sense of replications. eLife 6:e23383. 10.7554/eLife. 23383

Nuzzo R. 2015. Fooling ourselves. Nature 526:182-185.

Oakes M. 1986. Statistical inference: Commentary for the social and behavioural sciences. Chichester: Wiley.

Open Science Collaboration. 2015. Estimating the reproducibility of psychological science.

Orlitzky M. 2012. How can significance tests be deinstitutionalized? Organizational Research Methods 15:199-228. 10.1177/1094428111428356

Parker TH, Forstmeier W, Koricheva J, Fidler F, Hadfield JD, Chee YE, Kelly CD, Gurevitch J, Nakagawa S. 2016. Transparency in ecology and evolution: Real problems, real solutions. Trends in Ecology \& Evolution 31:711-719. 10.1016/j.tree.2016.07.002 
1477 Pericchi L, Pereira CAB, Perez M-E. 2014. Adaptive revised standards for statistical evidence. Proceedings of the National Academy of Sciences of the United States of America 111:E1935-E1935. 10.1073/pnas.1322191111

Poitevineau J, Lecoutre B. 2001. Interpretation of significance levels by psychological researchers: The .05 cliff effect may be overstated. Psychonomic Bulletin \& Review 8:847-850. 10.3758/bf03196227

Pritschet L, Powell D, Horne Z. 2016. Marginally significant effects as evidence for hypotheses: Changing attitudes over four decades. Psychological Science 27:1036-1042. $10.1177 / 0956797616645672$

Reinhart A. 2015. Statistics done wrong. San Francisco: No Starch Press.

Rosenthal R. 1979. The "file drawer problem" and tolerance for null results. Psychological Bulletin 86:638-641. 10.1037//0033-2909.86.3.638

Rosnow RL, Rosenthal R. 1989. Statistical procedures and the justification of knowledge in psychological science. American Psychologist 44:1276-1284. 10.1037//0003066x.44.10.1276

Rothman KJ. 2014. Six persistent research misconceptions. Journal of General Internal Medicine 29:1060-1064. 10.1007/s11606-013-2755-z

Rozeboom WW. 1960. The fallacy of the null-hypothesis significance test. Psychological Bulletin 57:416-428. 10.1037/h0042040

Sackrowitz H, Samuel-Cahn E. 1999. P values as random variables - Expected P values. American Statistician 53:326-331. 10.2307/2686051

Salsburg D. 2001. The lady tasting tea: How statistics revolutionized science in the twentieth century. New York: Henry Holt \& Co.

Sauley KS, Bedeian AG. 1989. .05: A case of the tail wagging the distribution. Journal of Management 15:335-344. 10.1177/014920638901500209

1502 Savalei V, Dunn E. 2015. Is the call to abandon p-values the red herring of the replicability crisis? Frontiers in Psychology 6:245. 10.3389/fpsyg.2015.00245

1504 Savitz DA. 2013. Reconciling theory and practice - What is to be done with P values? Epidemiology 24:212-214. 10.1097/EDE.0b013e318281e856 
1506 Schatz P, Jay KA, McComb J, McLaughlin JR. 2005. Misuse of statistical tests in Archives of

1507

1508

1509

1510

1511

1512

1513

1514

1515

1516

1517

1518

1519

1520

1521

1522

1523

1524

1525

1526

1527

1528

1529

1530

1531

1532

1533

1534

1535

1536 Clinical Neuropsychology publications. Archives of Clinical Neuropsychology 20:10531059. 10.1016/j.acn.2005.06.006

Schmidt FL. 1992. What do data really mean? Research findings, meta-analysis, and cumulative knowledge in psychology. American Psychologist 47:1173-1181. 10.1037/0003066x.47.10.1173

Schmidt FL. 1996. Statistical significance testing and cumulative knowledge in psychology: Implications for training of researchers. Psychological Methods 1:115-129. 10.1037//1082-989x.1.2.115

Schneider JW. 2015. Null hypothesis significance tests. A mix-up of two different theories: the basis for widespread confusion and numerous misinterpretations. Scientometrics 102:411-432. 10.1007/s11192-014-1251-5

Sedlmeier P, Gigerenzer G. 1989. Do studies of statistical power have an effect on the power of studies? Psychological Bulletin 105:309-316. 10.1037//0033-2909.105.2.309

Sellke T, Bayarri MJ, Berger JO. 2001. Calibration of $p$ values for testing precise null hypotheses. American Statistician 55:62-71. 10.1198/000313001300339950

Senn S. 2002. A comment on replication, p-values and evidence. Statistics in Medicine 21:24372444. $10.1002 / \operatorname{sim} .1072$

Sharpe D. 2013. Why the resistance to statistical innovations? Bridging the communication gap. Psychological Methods 18:572-582. 10.1037/a0034177

Simmons JP, Nelson LD, Simonsohn U. 2011. False-positive psychology: Undisclosed flexibility in data collection and analysis allows presenting anything as significant. Psychological Science 22:1359-1366. 10.1177/0956797611417632

Simonsohn U. 2014. Posterior-hacking: Selective reporting invalidates Bayesian results also. Available at https://papers.ssrn.com/sol3/papers.cfm?abstract_id=2374040 (accessed 6 June 2017)

Simonsohn U. 2015. Small telescopes: Detectability and the evaluation of replication results. Psychological Science 26:559-569. 10.1177/0956797614567341

Siontis KCM, Evangelou E, Ioannidis JPA. 2011. Magnitude of effects in clinical trials published in high-impact general medical journals. International Journal of Epidemiology 40:1280-1291. 10.1093/ije/dyr095 
1537 Skipper JK, Guenther AL, Nass G. 1967. The sacredness of .05: A note concerning the uses of

1538

1539

1540

1541

1542

1543

1544

1545

1546

1547

1548

1549

1550

1551

1552

1553

1554

1555

1556

1557

1558

1559

1560

1561

1562

1563

1564

1565 Tukey JW. 1991. The philosophy of multiple comparisons. Statistical Science 6:100-116.

Smaldino PE, McElreath R. 2016. The natural selection of bad science. Royal Society Open Science 3:160384. 10.1098/rsos. 160384

Song F, Parekh S, Hooper L, Loke YK, Ryder J, Sutton AJ, Hing C, Kwok CS, Pang C, Harvey I. 2010. Dissemination and publication of research findings: An updated review of related biases. Health Technology Assessment 14. 10.3310/hta14080

Stahel WA. 2016. Statistical issues in reproducibility. In: Atmanspacher H, Maasen S, eds. Reproducibility: Principles, problems, practices, and prospects: Wiley, 87-114.

Stanley DJ, Spence JR. 2014. Expectations for replications: Are yours realistic? Perspectives on Psychological Science 9:305-318. 10.1177/1745691614528518

Sterling TD. 1959. Publication decisions and their possible effects on inferences drawn from tests of significance - or vice versa. Journal of the American Statistical Association 54:30-34. 10.2307/2282137

Sterne JAC, Smith GD. 2001. Sifting the evidence - what's wrong with significance tests? British Medical Journal 322:226-231. 10.1136/bmj.322.7280.226

Stoehr AM. 1999. Are significance thresholds appropriate for the study of animal behaviour? Animal Behaviour 57:F22-F25. 10.1006/anbe.1998.1016

Thompson B. 1998. In praise of brilliance: Where that praise really belongs. American Psychologist 53:799-800. 10.1037//0003-066x.53.7.799

Thompson B. 1999. Why "encouraging" effect size reporting is not working: The etiology of researcher resistance to changing practices. Journal of Psychology 133:133-140.

Trafimow D, Marks M. 2015. Editorial. Basic and Applied Social Psychology 37:1-2. 10.1080/01973533.2015.1012991

Tryon WW. 2001. Evaluating statistical difference, equivalence, and indeterminacy using inferential confidence intervals: An integrated alternative method of conducting null hypothesis statistical tests. Psychological Methods 6:371-386. 10.1037//1082989x.6.4.371

statistical levels of significance in social science. American Sociologist 2:16-18.

Peer] reviewing PDF | (2017:03:16890:1:1:NEW 13 Jun 2017) 
1566 van Assen MALM, van Aert RCM, Nuijten MB, Wicherts JM. 2014. Why publishing everything is more effective than selective publishing of statistically significant results. PLoS One 9:e84896. 10.1371/journal.pone.0084896

1569

van Helden J. 2016. Confidence intervals are no salvation from the alleged fickleness of the P value. Nature Methods 13:605-606. 10.1038/nmeth.3932

Vankov I, Bowers J, Munafo MR. 2014. On the persistence of low power in psychological science. Quarterly Journal of Experimental Psychology 67:1037-1040. $10.1080 / 17470218.2014 .885986$

Whittingham MJ, Stephens PA, Bradbury RB, Freckleton RP. 2006. Why do we still use

Wasserstein RL, Lazar NA. 2016. The ASA's statement on p-values: Context, process, and purpose. The American Statistician 70:129-133. 10.1080/00031305.2016.1154108

Weinberg CR. 2001. It's time to rehabilitate the p-value. Epidemiology 12:288-290. 10.1097/00001648-200105000-00004

Weiss KM. 2011. The 5\% solution - How do we make decisions in science? Evolutionary Anthropology 20:81-84. 10.1002/evan20304

Wolf IS. 1961. Perspectives in psychology - XVI. Negative findings. Psychological Record 11:91-95.

Young NS, Ioannidis JPA, Al-Ubaydi O. 2008. Why current publication practices may distort science. PLoS Medicine 5:e201. 10.1371/journal.pmed.0050201

Yu EC, Sprenger AM, Thomas RP, Dougherty MR. 2014. When decision heuristics and science collide. Psychonomic Bulletin \& Review 21:268-282. 10.3758/s13423-013-0495-z

Ziliak ST, McCloskey DN. 2008. The cult of statistical significance: How the standard error costs us jobs, justice, and lives. Ann Arbor: University of Michigan Press. from case-control data. American Journal of Human Genetics 80:605-615. 